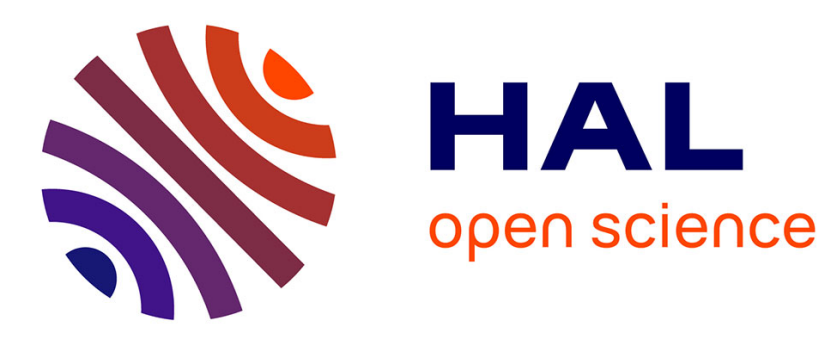

\title{
Les grands récits du paysage occidental. Une traversée historique et critique (xixe-xxie siècles)
}

\author{
Serge Briffaud
}

\section{To cite this version:}

Serge Briffaud. Les grands récits du paysage occidental. Une traversée historique et critique (xixexxie siècles). L'Information géographique, 2014, Paysage, 78 (3), pp.42-79. 10.3917/lig.783.0042 . halshs-01188468

\section{HAL Id: halshs-01188468 \\ https://shs.hal.science/halshs-01188468}

Submitted on 30 Aug 2015

HAL is a multi-disciplinary open access archive for the deposit and dissemination of scientific research documents, whether they are published or not. The documents may come from teaching and research institutions in France or abroad, or from public or private research centers.
L'archive ouverte pluridisciplinaire HAL, est destinée au dépôt et à la diffusion de documents scientifiques de niveau recherche, publiés ou non, émanant des établissements d'enseignement et de recherche français ou étrangers, des laboratoires publics ou privés. 


\title{
LES GRANDS RÉCITS DU PAYSAGE OCCIDENTAL Une traversée historique et critique (XIXè-XXIè siècle)
}

\author{
Serge BRIFFAUD \\ ADESS - UMR $5185 \mathrm{du}$ CNRS \\ CEPAGE (Centre de recherche sur l'histoire et la culture du paysage - École nationale supérieure \\ d'architecture et de paysage de Bordeaux)
}

Au cœur des spéculations dont la question paysagère a fait l'objet ces dernières décennies dans le domaine scientifique et philosophique, il y a l'idée, défendue par certains théoriciens, que le paysage n'est pas un simple donné perceptible, de tout temps et partout à portée de regard, mais plutôt une forme spécifique d'appréhension de « la nature » et du monde environnant — « a way of seeing » (Cosgrove, 1984) — qui serait propre à une période de l'histoire, à certains mondes sociaux et à certaines aires culturelles. La promotion de cette conception du paysage - il convient d'insister sur ce point - ne correspond pas à une simple revalorisation de ses dimensions symboliques et imaginaires, réagissant à une certaine focalisation des scientifiques — des géographes notamment, mais aussi des historiens et aujourd'hui des landscape ecologists sur sa dimension "matérielle » ou biophysique. Ce que porte ce paysage assimilé à une forme de regard, c'est la question même des modalités de l'identification du monde environnant, posée du point de vue de leur hétérogénéité sociale, culturelle et historique. Les enjeux épistémologiques sous-jacents à cette appréhension de la notion de paysage apparaissent, ainsi, particulièrement prégnants. Ils n'ont trait à rien moins qu'à la construction/reconstruction de l'identité et des interfaces de certaines disciplines des sciences humaines et sociales. Cela est vrai pour la géographie, mais aussi, quoique parfois dans une moindre mesure, pour d'autres disciplines concernées par cette même idée du paysage: histoire de l'art, anthropologie, philosophie esthétique... Mais ces enjeux sont aussi, et inséparablement, d'ordre politiques et sociétaux, à l'heure où la notion de paysage sert d'étendard à certaines conceptions alternatives de l'aménagement, de la gestion, de l'urbanisation et de la patrimonialisation des territoires (Luginbühl, 2007 et 2012).

Les lignes qui suivent proposent une exploration de cette conception du paysage à partir d'un point de vue particulier : celui des discours qu'elle a générés, et dont elle apparaît de fait inséparable, sur les « origines » du paysage, c'est-à-dire sur le moment - mais cette question du moment est indissociable de celle du lieu - où émerge cette forme de regard sur le monde environnant. Situer ce moment de l' « invention » du paysage - pour reprendre une expression dont différents auteurs ont fait à ce propos usage — c'est prouver l'irréductible 
spécificité de cette perception et la départir de cette universalité et de cette évidence qu'on lui a inconsidérément attribué. C'est, ainsi, légitimer par un recours à la preuve historique une réforme du concept et son assignation à de nouveaux usages ; mais ce peut être là aussi, et à l'inverse — on va le voir — le moyen de régénérer une conception héritée du paysage. Dans un cas comme dans l'autre, les théories sur l'origine du paysage qui ont fleuri depuis trois à quatre décennies tiennent donc bien du discours de (re)fondation. Elles prennent la forme de grands récits du paysage occidental, dans la mesure où toutes, sans exception, attribuent à l'Occident la paternité de la perception paysagère du monde.

Or, si cette archéologie des commencements apparaît intimement liée au discours théorique de ces trois dernières décennies en matière de paysage, elle ne constitue pas, considérée à l'échelle de la longue durée historique un point de vue original, ni nouveau sur la question. Au fondement des lignes qui suivent, il y a le constat de l'ancienneté et de la récurrence du recours à ces grands récits de fondation. La quête des origines d'une appréhension paysagère du monde environnant apparaît ainsi en réalité consubstantielle au discours occidental sur le paysage depuis environ deux siècles. C'est dans la première moitié du XIXè siècle que l'on voit apparaître cette manière de «construire » le paysage en proposant des scénarios de son avènement et en énonçant, dans le même mouvement, les conditions qui lui permettent d'exister comme regard, sentiment ou représentation spécifiques. La production théorique en la matière prend déjà alors la forme de récits qui, tout en disant les origines, regardent vers le présent et le futur, et visent à associer la caution de l'histoire à une codification des usages contemporains de la notion. Passé le milieu du XIXè siècle, quelques auteurs reprennent ensuite à leur compte cette démarche, ici et là, à différents moments, avant qu'elle ne se réinvite, à partir des années 1980, au cœur même des théories paysagères et, plus largement, du discours en la matière. Entre les deux grands moments du récit paysager « originiste ", une coupure très nette apparaît toutefois. Les modernes ne lisent pas, en un mot, les anciens. Ils n'ont pas conscience de s'inscrire dans un champ multiséculaire de spéculation, voire de proposer des interprétations depuis longtemps avancées.

Les discours contemporains sur les origines du paysage mérite ainsi d'être interrogés comme réémergence et comme actualisation. Cette perspective permet d'élargir le point de vue critique développé à l'égard des grands récits paysagers d'aujourd'hui par des auteurs qui se sont employés à déconstruire, en même temps que les scénarios historiques proposés, l'idée du paysage qu'ils accréditent. Dans le monde anglo-saxon, W.J.T. Mitchell, notamment, s'est livré à un examen sans concession de ces scénarios téléologiquement ordonnés autour de l'idée d'une " conquête " occidentale du paysage, montrant comment ils ont masqué et consolidé à la fois la dimension "impériale » et "impérialiste » du paysage (Mitchell, 1994a). Hervé Brunon a donné en 2006 une vision très complète et très éclairante des thèses avancées dans la seconde moitié du XXème siècle par les historiens de l'art sur «L'essor artistique et la fabrique culturelle du paysage à la Renaissance », et il en a construit une critique complétant très utilement celle qu'avait proposée en 1998 l'auteur de ces lignes. Reprenant ce dossier en 2011, Brunon a mis à jour les enjeux épistémologiques de la question paysagère pour l'histoire de l'art contemporaine (Brunon, 2006 et 2011). D'autres encore se sont 
employés à la déconstruction de ce que Michael Jakob appelle la « success story paradigmatique et typiquement occidentale » du paysage (Jakob, 2001, p. 9). L'exploration des soubassements de ces grands récits du paysage occidental mérite néanmoins d'être poussée plus loin encore. Le changement d'échelle temporelle proposée ici peut y aider, en restituant sa dimension pluriséculaire à la problématique de l'« invention » du paysage et aux discours qui la prennent en charge. Il fait apparaître ces derniers comme des sources particulièrement éclairantes sur les enjeux à la fois épistémologiques, philosophiques et politiques sous-jacents, durant les deux derniers siècles, aux spéculations théoriques sur le paysage, et, plus globalement, on va le voir, sur le rôle joué par la question paysagère dans les définitions et redéfinitions contemporaines du rapport de l'humanité et de la société à la nature. Enfin, élargir ainsi la focale historique aide non seulement à construire la critique des théories les plus contemporaines sur les origines du paysage, mais aussi à dépasser cette approche et à proposer d'autres manières d'aborder, à la fois, l'histoire du paysage occidental et la construction de cette notion de paysage elle-même.

\section{LA PROBLÉMATIQUE DE L'ÉMERGENCE DU PAYSAGE À L'AUBE DE L'ÉPOQUE CONTEMPORAINE}

Dans la première moitié du $\mathrm{XIX}^{\circ}$ siècle commence à se poser la question, qui demeure aujourd'hui souvent au cœur des discours sur l'émergence du paysage en Occident, des origines picturales du paysage, c'est-à-dire du moment et des conditions dans lesquels une représentation paysagère du monde a pu s'imposer. Dès cette période, l'avènement du paysage dans l'art est perçu comme la marque d'un tournant majeur dans l'histoire des sensibilités humaines et du rapport homme/nature, la peinture n'apparaissant ainsi que comme le symptôme et le vecteur privilégiés d'un phénomène qui la dépasse. Des travaux récents sur l'histoire des théories picturales de l'époque romantique ont montré comment cette question de l'invention du paysage a alors été prise en charge par des peintres et des philosophes de l'art (John Constable, William Turner, Philipp-Otto Runge, Wilhelm Schlegel...) ', mais aussi par un savant tel qu'Alexandre de Humboldt, que l'on peut considérer comme l'auteur de la théorie historique la plus élaborée produite à cette époque sur les origines du paysage (Humboldt, 2000 [1846-1851]; Recht, 1989; Briffaud, 2006). Enjeux épistémologiques et artistiques se croisent, à l'époque romantique, en cette problématique autour de laquelle on voit se dessiner une certaine vision de la vision, c'est-à-dire du statut de l'observateur confronté aux spectacles de la nature et des pouvoirs de l'œil dans son rapport à l'environnement perceptible.

\footnotetext{
1 . Les lignes qui suivent s'appuient largement et notamment sur: DECULTOT (1996) et WAT (1998).
} 


\section{Subjectivisation du paysage et épuration du champ visuel}

Le problème des origines et des développements de la peinture de paysage apparait à ce moment jouer le rôle de discours de légitimation pour un mouvement pictural qui tend à placer le paysage au sommet de la création artistique, subvertissant ainsi un ordre néo-classique des respectabilités académiques qui opposait l'idéal porté par la peinture d'histoire à l'imitation servile et plate de la réalité perceptible, par laquelle le peintre paysagiste serait toujours menacé (Lee, 1991). C'est en Allemagne que l'effervescence théorique autour de ces questions fut la plus vive et que la question du paysage revêtit le plus nettement un caractère subversif, sur fond d'émergence des principes de l'art romantique et de la Naturphilosophie, mais aussi en liaison avec l'essor d'un sentiment national qui fait du paysage, comme l'a montré François Walter, un emblème privilégié de l'identité germanique (Walter, 2004 ; Decultot, 1996).

Mais dans ce débat, le paysage n'est pas regardé comme un simple genre de peinture. Si la question de son émergence prend un telle importance, c'est parce qu'on le considère comme la forme de perception et de sentiment qui caractérise un homme nouveau, destiné à renouer avec une nature dont on avait perdu de vue l'évidence sensible et la simple vérité, en s'égarant dans ce que John Constable appelle «les champs vides de l'idéalisme » ${ }^{2}$. Tel qu'il se présente, surtout, en Allemagne, le discours sur les origines du paysage apparaît essentiellement prospectif et programmatique. Il vise un art de peindre, une science, une manière d'être et de sentir qui appartient moins au passé qu'au futur, c'est-à-dire non encore vraiment advenue, ou en voie seulement de l'être. Évoquer l'avènement du paysage revient ainsi à décrire un processus dont on ne saisirait, dans le passé comme dans le présent, que les prémices. Le discours historique, autour de cette thématique, se confond en un mot avec un discours prophétique.

Ce qui ressort, globalement, de ces premiers grands récits de fondation du paysage, est que l'appréhension paysagère du monde s'enracinerait dans le mouvement duel d'un divorce avec la nature et d'une nouvelle union avec elle. Pour A.W. Schlegel, c'est précisément la distance irréductible qui sépare les populations urbanisées de la véritable nature qui les a conduit à se tourner vers le paysage comme vers un succédané de cette dernière. Réduit à une "nature portative », le paysage permet alors de profiter des avantages de la nature sans en supporter les inconvénients. Longtemps la peinture de paysage fut ainsi associée, selon Schlegel, à une vision frivole et utilitaire de la nature. Loin de favoriser la découverte de cette nature, sa mise à distance par les hommes a donné naissance à la vision mutilante et aseptisée rendue par les peintres de paysages. Dans un premier temps donc, le paysage ne fut que l'un des symptômes d'une crise du regard. Reprenant un thème qui traverse tout le XVIII ${ }^{\circ}$ siècle, Schlegel dénonce, en 1799, cette incapacité de l'homme à voir «au nom de la vue »: «... fondamentalement, la conscience de ce qui nous entoure, lorsqu'il s'agit d'objets courants, nous vient davantage de ce que nous savons que de ce que nous voyons. ${ }^{3}$. Le champ visuel est encombré par des concepts qui masquent

\footnotetext{
2. Cité par P. WAT (1998, p. 77).

3 . A.W. SCHLEGEL, Les Tableaux (1799), cité par R. RECHT. 1989. La Lettre de Humboldt... Ouvr. Cité, p. 20.
} 
l'évidence. Il faut donc, pour Schlegel, réapprendre à voir. Et qui peut réenseigner cet art? Le peintre de paysage lui-même, répond l'auteur des Tableaux et de l'Athenaeum; mais à la condition de ne plus se complaire dans l'insignifiant spectacle d'une natura naturata et de viser à atteindre la natura naturans, c'est-àdire de passer de la représentation de l'œuvre achevée à celle de la puissance productrice et créatrice. Or, pour Schlegel, rien dans le spectacle de la nature extérieure ne peut aider le peintre à atteindre cette autre nature. Les ressources sur lesquelles ce dernier peut compter résident exclusivement en son for intérieur et dans son propre pouvoir de créer. Schlegel, ne va pas toutefois jusqu'à déclarer l'autonomie de la peinture de paysage vis-à-vis de tout référent extérieur. En se tournant vers ses propres forces spirituelles, le peintre assume la condition de l'homme, qui est celle d'un microcosme reflétant le macrocosme. L'image que l'artiste produit de la nature, dès lors qu'il accepte d'en abandonner un temps la vision matérielle pour mieux se replier sur lui-même, est nécessairement en sympathie avec elle. Et c'est en cela précisément qu'en retour, elle peut réapprendre aux hommes à voir à nouveau ce spectacle de la nature auxquels ils demeuraient aveugles. L'artiste reprend ainsi à son compte cette rupture de la relation homme/nature. Il l'assume pour la transcender et la résoudre. Et c'est à la fois ce vide et ce plein, cette prise de distance et cette réconciliation, qu'il revient au paysage, objet empreint d'une tension irréductible, de porter et d'exalter dans un art neuf.

C'est relativement à cette vision de l'art du paysage que prend tout son sens l'affirmation bien connue de Schlegel, que l'on trouve dans ses Leçons sur la littérature et les Beaux-Arts (1801): "Le paysage en tant que tel n'existe que dans l'œil de son spectateur ${ }^{4}$. Il faut comprendre ici que le paysage est la forme que revêt cette nature vers laquelle on fait retour après en avoir été séparé - une nature qui n'est pas l'invention d'une libre subjectivité, mais plutôt la forme que prend son spectacle aux yeux d'un observateur qui ne renoue avec elle, en tant qu'entité perceptible, qu'à la condition de l'éclairer d'une lumière venant de l'intérieur. Si cette phrase porte bien la mise en question d'une nature-objet, séparable de son observateur et qui pourrait être mise à l'abri des affects du sujet regardant, elle n'assigne donc pas pour autant le paysage à une subjectivité qui serait propre à l'artiste et que l'on pourrait opposer à l'objectivité du savant, mais contient plutôt implicitement un appel à un renouvellement de la rationalité savante elle-même.

La vision schlegelienne du paysage - mais on peut étendre la remarque à tous les discours de cette époque sur l'avènement des paysages — hérite des spéculations sur la question paysagère qui ont traversé les Siècle des Lumières. Elle réinvestit en particulier la thématique du pittoresque, à laquelle celle du paysage s'est trouvé liée, Outre-Manche, dès le début du XVIIIè siècle, à travers les spéculations sur la spécificité de la peinture dans le concert des arts et sur la réforme d'un art des jardins qui devient alors, sous la forme du landscape gardening, l'art de "composer les paysages » à la manière des peintres, mais aussi à travers les spéculations sur l'art de voir le monde à travers la peinture (Andrews, 1990 ; Hunt, 1992 ; Milani, 1996; Baridon, 2000). Schlegel, qui

4 . Cité par Elisabeth DECULTOT. 1996. Ouvr. cité, p. 177. 
propose une critique radicale du jardin pittoresque, reprend en revanche à son compte et développe cette idée d'une vision pittoresque, qui se présente comme la condition permettant aux créations picturales de faire retour vers l'environnement perceptible pour, d'une certaine manière, prendre sa place et lui permettre, dans le même mouvement, de porter l'image de la natura naturans. C'est à cette parfaite interchangeabilité de l'œuvre d'art et du réel perçu que doit mener le développement futur d'un art véritable du paysage.

En posant ce cadre de raisonnement et en fixant ainsi les enjeux d'une spéculation sur l'invention — ou plutôt, donc — la réinvention du paysage, Schlegel participe largement à fixer les fondements de l'exhaussement de la question paysagère dans le débat artistique et culturel de l'époque romantique. Il est suivi dans la voie qu'il a tracé par de nombreux auteurs dans les théories desquels reviennent en permanence quelques principes fondamentaux. L'idée d'un paysage remédiant au divorce initial du sujet et de la nature, mais aussi et surtout celle du nécessaire "désencombrement» du champ visuel, se retrouve en particulier chez le peintre et théoricien de la peinture Philipp-Otto Runge, dont les théories sont contemporaines à celle de Schlegel. Pour Runge, le paysage est imprégné des significations que lui a communiquées l'homme, des sentiments qu'il a éprouvés et qui se sont à travers le temps comme incrustés dans les éléments naturels (Décultot, 2009). Pour ce défenseur de la «Landschafterey », il s'ensuit que représenter le paysage permet de représenter l'homme lui-même et n'est donc en rien contradictoire avec le précepte majeur énoncé par les théoriciens néo-classiques de la peinture, qui enjoignaient l'artiste de se focaliser sur la figure humaine, en pratiquant de préférence la peinture d'Histoire et accessoirement le portrait. Le basculement ainsi (ou autrement) justifié des priorités picturales, qui revient en réalité à une fusion des genres de peinture dans le paysage, est, pour Runge comme pour beaucoup d'autres après lui, la marque de l'entrée dans un temps nouveau, qui est celui d'une immédiateté retrouvée du rapport de l'homme à une nature désormais dégagée des lourdes allégories sous lesquelles on l'avait enfouie, et redevenue ainsi loquace. C'est par conséquence, chez Runge aussi, un "nettoyage » de l'espace de la représentation et de la perception elle-même qui permet à la nature d'exister comme spectacle paysager, processus qui correspond, dans la conceptualisation gœthéenne du langage artistique, au passage du règne de l'allégorie à celui du symbole.

\section{L'avènement du paysage selon $A$. de Humboldt}

Le premier scientifique à avoir fait sienne la problématique de l'avènement du paysage semble être Alexandre de Humboldt, qui place ce questionnement au cœur du long exposé qu'il consacre, dans son ultime ouvrage, à l'histoire du sentiment du « cosmos » (Humboldt, [1846-1851] 2000 ; Recht, 1989 ; Briffaud, 2006). L'essai humboldtien se situe dans la continuité directe des spéculations proposées à l'époque romantique, à travers le prisme de l'histoire de l'art, sur cette même question. On peut considérer le savant allemand comme l'un de ceux qui ont contribué à fixer l'archétype discursif de l'autonomisation du paysage dans les œuvres, Humboldt se consacrant, pour y évaluer la place donnée à une représentation de la nature et du sentiment qu'elle suscite, à un long balayage de 
l'histoire de la peinture et de la poésie depuis les origines de l'humanité. Dans l'introduction de Cosmos, Humboldt s'emploie à décrire le processus de dessillement qui a permis à l'homme occidental de précisément être en mesure de percevoir et d'éprouver, dans le spectacle concret du monde qui l'entoure, une nature-cosmos, c'est-à-dire — et l'on retrouve ici Schlegel — le spectacle enthousiasmant et magnifique dans lequel se reflète les lois qui organisent ce que le savant allemand appelle la "connexité » des phénomènes naturels. Ce processus là correspond au passage d'un sentiment vague et abstrait de l'unité de la nature, qui a existé depuis les origines de l'humanité, à un sentiment qui se nourrit de la matérialité concrète du paysage perceptible. C'est cette concrétisation du sentiment qui se produit dans ce que Humboldt présente comme le premier acte du processus qui conduit à l'émergence d'un sentiment paysager de la nature ; à savoir à ce moment où les Européens se trouvent pour la première fois confrontés, à l'aube des temps modernes, au grand spectacle cosmique des Andes tropicales. Pour la première fois alors a en effet été donnée à cette partie de l'humanité, selon l'auteur de Cosmos, la possibilité de percevoir directement, en un seul et même paysage, et en un seul et même coup d'œil, l'effet des lois qui déterminent la répartition des éléments naturels sur l'ensemble de la planète.

L'essai de Humboldt est d'abord sous-tendu par la volonté de tirer du paysage un parti épistémologique et de montrer son avènement comme un palier décisif franchi en direction d'une science aux modes d'objectivation profondément renouvelés. Les thèses proposées dans Cosmos sur la naissance du sentiment paysager de la nature apparaissent consubstantielle à ce moment, analysé par Michel Foucault (1966), où la science essentiellement descriptive que constitue l'Histoire naturelle cède le pas, sur fond d'individualisation des disciplines, à une science visant la compréhension des fonctionnements de la nature et des lois qui les régissent. La causalité dont cette science part en quête a pour foyer principal une histoire de la nature désormais comprise non plus comme la description analytique de son aspect extérieur, mais comme le scénario de ses transformations.

Or, c'est dans la mise en crise de ce rapport au visible que Humboldt voit précisément la principale source de l'avènement d'une vision paysagère de la nature. Le paysage se dévoile, pour lui, à l'homme occidental dans le mouvement d'une remontée vers la surface d'un regard qui a pris le risque de sa propre négation en visant l'élucidation des fonctionnements " profonds » de la nature. Si ce dévoilement a pu être le fait, comme pour les colons découvrant les Andes tropicales, de circonstances exceptionnelles de la perception, il est surtout le résultat de l'accession à ce qu'il conviendrait de nommer une seconde vue - une vue qui n'advient, comme ce qu'elle dévoile, qu'au retour d'une plongée dans l'histoire de la nature. L'avènement du paysage renvoie ainsi, pour l'auteur de Cosmos, à la capacité nouvelle acquise par l'homme occidental — et par lui seulement - grâce à une certaine science, à saisir dans les spectacles perceptibles de la terre l'action des lois constantes de la nature, celles-ci s'exprimant dans les «connexions » solidarisant des éléments naturels que les savants, naguère, isolaient et se contentaient de décrire. Le «temps du paysage » - l'entrée de l'humanité occidentale dans cette nouvelle forme de rapport à la nature - est aussi celui où peut s'opérer l'inscription d'une science renouvelée dans le regard 
et les expériences sensibles de l'homme ordinaire, confronté au quotidien à une nature s'offrant désormais à être, dans un même mouvement, ressentie et décryptée. Il est celui où devient concevable une géo-graphie qui disposerait les hommes à saisir ce qui est écrit à la surface du monde et à construire de celui-ci une « vue raisonnée ».

L'idée du paysage qui se dégage des thèses de Humboldt peut être notamment rapprochée des thèses énoncées quelques années plus tôt par CarlGustav Carus, médecin et peintre, défenseur d'un art de la représentation de la vie de la terre (Erdlebenbildkunst) et d'une physiognomonie du paysage par laquelle la peinture se met au service de la science en s'imposant comme l'instrument d'une mise en relation des profondeurs et des surfaces (Carus et Friedrich, [1831] 2003 ; Decultot, 1996 ; Deligne, 2006). Chez Carus comme chez Humboldt, l'ère nouvelle qui s'ouvre pour l'art et la science est liée au défi que représente l'appréhension d'une nature en mouvement, travaillée par une histoire qui institue le paysage comme trace et appelle au mariage de la contemplation et de l'exploration. Faire revenir à la surface d'un paysage où elle ne fait qu'affleurer cette vérité " profonde » de la nature - c'est-à-dire, pour Carus, sa puissance vitale - revient pour le peintre à renouer avec le sens premier du mot représentation et la fonction primitive de l'image : redonner vie, " présentifier l'absent, comme si ce qui revenait était le même et parfois mieux, plus intense, plus fort que si c'était le même » (Marin, 1993, p.11).

\section{DISTANCIATION PAYSAGÈRE ET REGARD PICTURAL}

L'époque romantique n'a pas seulement inauguré la forme du «grand récit» originiste appliqué au paysage. Elle a proposé une interprétation de l'avènement de cette forme de regard et de représentation dont bien des traits traversent le temps et forment l'armature des théories ultérieures, y compris les plus contemporaines. Il en est ainsi, en particulier, de l'idée que le paysage émerge dans le mouvement d'un retour vers une nature avec laquelle on a pris ses distances, voire de laquelle on a divorcé. On retrouve plus tard cette idée, par exemple, chez un Norbert Elias, qui évoque l'attrait irrésistible qu'exercent la campagne, au début du XVIIIème siècle, pour des aristocrates de cour contraints depuis longtemps à s'éloigner de leurs domaines ruraux et qui ont enfin la possibilité, le carcan curial se desserrant, de revenir vers eux. Ce qui était, avant le règne de Louis XIV, un espace simplement vécu au quotidien, est ainsi devenu pour cette noblesse déracinée, constate Elias, un paysage sur lequel on projette des rêves nourris par les poètes et peintres de l'idylle et de l'églogue. Le lieu naguère familier s'est métamorphosé en un spectacle à contempler (Elias, [1933] 1985).

Cette même idée avait auparavant été magistralement développée au début du XXème siècle, dans un texte aussi remarquable que méconnu, par le poète Rainer Maria Rilke, chez qui on la trouve étroitement associée au scénario de l'émergence d'une peinture de paysage. La distanciation au fondement de la vision paysagère prend ici la forme d'un divorce tragique, qu'il revient à l'artiste de sublimer : 
«Et il fallait regarder le paysage comme une chose lointaine et étrangère, comme une chose perdue et sans amour, qui s'accomplit tout entière en ellemême, afin qu'il pût servir un jour de moyen et de point de départ à un art autonome. Il fallait qu'il fût loin et très différent de nous afin de pouvoir devenir une parabole libératrice de notre destin. Il fallait que dans son indifférence sublime il se montrât presque hostile pour pouvoir offrir à notre existence une nouvelle interprétation grâce à ses objets. Et c'est dans cet esprit qu'a pris forme cet art du paysage dont Léonard de Vinci avait déjà eu le pressentiment et la maitrise. Il se développa lentement, entre les mains de solitaires, de siècle en siècle. Longue était la route qu'il fallait suivre car il était difficile de se désaccoutumer du monde assez complètement pour ne plus le voir avec l'œil prévenu de l'indigène qui rapporte tout à lui-même et à ses besoins lorsqu'il regarde. Il faut souvent que quelqu'un vienne pour nous dire ce qui nous entoure; il fallut donc commencer par écarter de soi les choses pour devenir capable par la suite de s'approcher d'elles de façon plus équitable et plus sereine, avec moins de familiarité et avec un recul respectueux car on ne commençait à comprendre la nature qu'à l'instant où on ne la comprenait plus ; lorsqu'on sentait qu'elle était autre chose, cette réalité qui ne prend pas part, qui n'a point de sens pour nous percevoir, ce n'est alors que l'on était sorti d'elle, solitaire, hors d'un monde désert.

«Et il fallait cela pour qu'on devint artiste par elle; il ne fallait plus l'éprouver en tant que sujet, dans la signification qu'elle avait pour nous, mais comme un objet, comme une grande réalité qui était là. ${ }^{5}$

C'est dans un semblable cadre d'interprétation, prolongeant celui qu'avait construit l'époque romantique, que prend corps tout un courant de la théorie contemporaine du paysage, essentiellement représenté par l'histoire de l'art et la philosophie esthétique. Au fondement de cette vision de l'avènement du paysage, il y a l'association étroite du sentiment auquel on l'assimile à sa "révélation » artistique et en particulier picturale. Il y a aussi un processus de distanciation qui peut se lire comme un procès d'épuration du regard, c'est-à-dire autonomisation de la perception à l'égard de toute détermination liée à la familiarité avec la chose perçue, au vécu qui unit à elle, ou à ce qu'on peut connaître d'elle par la médiation de savoirs constitués.

5 . RILKE, 1966, p. 372. Il faut ajouter que contrairement à un A.W. Schlegel par exemple, dont il sera question au chapitre 3, Rilke ne reconnaît entre l'homme et la nature aucune forme de sympathie susceptible de s'inscrire dans une relation microcosme/macrocosme. Dans sa version post-darwinienne, l'hypothèse d'une absolue séparation de l'homme et de la nature se nourrit de fantasmes anxiogènes : "Sans doute, quelqu'un d'entre nous pourrait-il évoquer notre parenté avec la nature, dont nous descendons, comme les derniers fruits d'un grand arbre généalogique qui s'élève au-dessus de nous ; mais ce faisant, on ne saurait nier que cet arbre, lorsque nous remontons branche après branche et rameau après rameau, ne tarde pas à se perdre dans les ténèbres; en des ténèbres qu'habitent des animaux géants et disparus, des monstres d'hostilité et de haine, et que, plus nous remontons en arrière, plus nous nous approchons d'êtres de plus en plus étrangers et cruels, de sorte que nous sommes obligés de supposer que nous trouverions la nature comme la réalité la plus cruelle et la plus étrangère de toutes. » (Ibid., 375). 


\section{Le paradigme gombrichien}

C'est dans l'essai que l'historien de l'art Ernst Gombrich consacre, en 1953, au rapport entre essor du paysage et théorie artistique à la Renaissance qu'il faut chercher le texte de référence majeur auxquels s'alimenteront les spéculations contemporaines sur les origines de l'image et du sentiment paysagers (Gombrich, 1953). Ce texte présente le scénario, très largement repris par la suite, de l'émergence d'un «pur» paysage peint, dont est posée à la fois l'existence et la pertinence en tant qu'objet historique majeur(Brunon, 2006). L'essai de Gombrich contribue largement à promouvoir l'idée, déjà présente chez Rilke et chez les théoriciens de l'époque romantique, que la représentation picturale est antérieure à l'appréciation esthétique du spectacle de la nature in situ :

« ... alors que l'on considère d'habitude, écrit Gombrich, que la « découverte du monde » est le principal motif qui explique l'essor de la peinture de paysage, nous avons presque envie de renverser la formule et d'affirmer l'antériorité de la peinture sur le «sentiment» du paysage.»(Gombrich, [1953] 1983, p. 33)

Sur cette base, c'est au processus même qui a permis l'émergence d'une peinture «purement» paysagère que l'on a eu tendance à assimiler celui qui permet au sentiment du paysage lui-même d'émerger. Ce processus, qui concerne à la fois l'image et le regard, se présente comme un décapage. Le paysage ne serait ainsi représenté, selon Gombrich et ses émules, pour lui-même que lorsque l'image se libère de toute signification allégorique, des dogmes religieux cristallisés en codes iconographiques; de tout ce qui, en un mot, s'interposait entre le regard et les choses mêmes. Et c'est, pour employer les mots de Mitchell, ce même « mouvement progressif en direction d'une épuration du champ visuel », qui amènerait l'homme occidental à se saisir, dans son environnement même, de la « simple présence des choses » (Mitchell , 1994, p. 1). La Renaissance est ainsi présentée comme le moment clé d'un dessillement : celui où l'homme occidental se montre enfin capable, par la médiation de la peinture, de jouir du spectacle du monde dans lequel il vit.

Ce scénario est repris et vulgarisé en France par le philosophe Alain Roger, au début des années 1980, à l'appui de sa théorie de l'artialisation ${ }^{6}$. Par ce dernier terme, l'auteur du Court traité $d u$ paysage désigne une opération d'esthétisation: celle la même qui transforme en «paysage » le «pays » environnant. L'artialisation empaysage par un double voie : celle, d'un côté, de la création littéraire, mais surtout iconographique et picturale («artialisation in $v i s u »)$, produisant des œuvres qui fonctionnent comme des prismes grâce auxquels peut s'opérer une esthétisation de la perception du monde extérieur; et celle, d'un autre côté de la création jardinière, qui est action directe sur le «pays » (« artialisation in situ»). Roger reprend en outre l'idée que cet empaysagement du monde est datable et consacre de longs développements à l'exposé des conditions de possibilités historiques d'une telle perception du monde extérieur. Il fait également sienne l'idée qu'elles ont été rassemblées à la Renaissance, avec les premières représentations artistiques du paysage produites, au XVème siècle, par

\footnotetext{
6 . Les thèses d'Alain Roger sur le paysage sont résumés dans son ouvrage : A. ROGER. 1997. Court traité du paysage. Paris : Gallimard.
} 
les peintres flamands, la médiation des auvres picturales habilitant dès lors le paysage à devenir le terrain par excellence d'une " pure » expérience esthétique. Roger insiste sur l'idée que la laïcisation des esprits aurait été l'une des conditions majeures du paysage. La perspective géométrique de la Renaissance est présentée comme un outil de cette laïcisation, dans la mesure où elle aurait ouvert, au-delà des personnages et des scènes occupant le premier plan du tableau, un espace où le paysage se serait trouvé " à l'écart et comme à l'abri du sacré ", avant de devenir un objet de représentation se suffisant à lui-même ${ }^{7}$. On retrouve également ici l'idée, présente chez Rilke, de l'incapacité de l'«indigène » Roger remplace le terme par celui de " paysan ", mais cela revient au même - à éprouver ce qui l'entoure comme "paysage ». Cette même incapacité frappe le scientifique qui cherche à comprendre, l'avènement du paysage supposant un regard détaché et épuré, qui n'appréhende le pays ni comme ressource, ni comme objet de connaissance.

\section{Le scénario berquien}

Si le scénario proposé par Roger suppose le savant purement et simplement inapte au paysage, de nombreux autres auteurs fondent leurs théories sur le constat d'une homologie structurale unissant la distanciation « paysageante » à la distanciation objectivante par laquelle la science moderne se définit. Il revient à Humboldt, on l'a vu, de défendre le premier l'idée d'une implication du regard distancié du scientifique dans l' «empaysagement» du regard des Occidentaux sur la nature. Rilke qualifie lui-même d' " objective » la vision qui naît de ce processus d'épuration du champ visuel nécessaire à l'avènement du paysage, suggérant ce rapprochement avec la vision du savant, qui s'exclut lui-même par méthode de son objet et l'institue ainsi comme tel. Ce rapport entre distanciation scientifique et sentiment du paysage est aujourd'hui réexaminé par certains théoriciens, parmi lesquels Augustin Berque. D'une façon générale, les thèses de Berque sur l'émergence, en Occident, d'une vision paysagère du monde environnant s'inscrivent dans le paradigme gombrichien, présenté plus haut ${ }^{8}$. C'est en se transformant en représentation de lui-même que ce monde s'empaysage aux yeux de l'homme occidental. Berque apporte toutefois au scénario épiphanique traditionnel une nuance nouvelle en avançant que les peintres de la Renaissance préfigureraient, en faisant entrer le paysage dans leurs œuvres, l'objectivation scientifique de la nature, qui s'opèrera au temps de Galilée et de Descartes. Tout en constituant le sujet comme tel, le principe de la distanciation objectivante écarte la vérité de la nature du milieu dans lequel ce sujet évolue au quotidien. Le paysage, estime Berque, va être dès lors en quelque sorte amené à constituer, sinon l'antidote à la relation au monde dont sa propre

\footnotetext{
7 . «En instituant une véritable profondeur, [la perspective] met à distance ces éléments du futur paysage et, du même coup, les laïcise. Ils ne sont plus des satellites fixes, disposés autour des icônes centrales, ils forment l'arrière-plan de la scène (au lieu du fond doré de l'art byzantin), et c'est tout différent ; car là il se trouve à l'écart et comme à l'abri du sacré. Mais les voilà condamné à se forger une unité. »(A. ROGER. 1997, p. 70).

8 . Cette théorie est présentée, sous des formes parfois sensiblement différentes, dans de nombreuses publications. Cf. notamment : BERQUE, 1995 ; et surtout BERQUE, 2008.
} 
apparition annonce l'instauration, au moins l'autre face d'une nature dont la réalité devient irrémédiablement double, phénoménale et factuelle. Il deviendra la forme sensible et esthétique d'un rapport occidental à la nature dont la science moderne incarne la dimension rationnelle.

On peut associer à ce scénario épiphanique celui que présente Philippe Descola (2005, p. 91-99 et 2011), qui, situant lui-même à la Renaissance le moment de l'émergence du paysage, insiste tout particulièrement sur le rôle décisif de la Perspectiva artificialis, permettant alors au paysage de trouver sa place dans l'image et à cette dernière de devenir, au moins potentiellement, l'espace d'une "imitation de la nature » comprise comme représentation de la chose même, c'est-à-dire délivrée de toute signification religieuse et, plus généralement, de sa valeur de signe. Descola, reprenant Panofsky, met aussi et surtout en avant le paradoxe de la perspective, qui place un nouvel espace, continu, rationnel et mathématiquement construit, dans la dépendance du regard humain et de la subjectivité qui lui est inhérente. Le paysage apparaît ainsi à ses origines - et Descola rejoint Berque en particulier sur ce point — comme un dispositif de perception et de représentation de la nature qui reflète l'ambiguïté structurelle de sa mise à distance, celle-ci se présentant comme la condition de possibilité à la fois de l'objectivation scientifique et de l'appréciation subjective d'un monde devenu « extérieur ».

Chez Berque et Descola, ce récit de l'émergence du paysage apparaît indissociable de l'exhaussement de ce dernier au niveau d'un critère anthropologiquement discriminant, au sens où le paysage est appréhendé ici comme une forme distinctive de relation à l'étendue terrestre ou à la nature, permettant d'établir à cet égard des partages entre les sociétés à l'échelle planétaire. Berque, on le sait, parle de «sociétés paysagères » (la Chine et l'Occident), ou encore à propos du Moyen Âge européen, de «société protopaysagère ». Il dresse - mais je n'y reviens pas ici — une liste de «conditions " qui permettent de parler d'une relation "paysagère" de l'homme à son environnement (Berque, 1995). Pour A. Berque, le paysage est donc tout sauf un simple regard plus ou moins contemplatif et esthétisant porté par l'homme sur le monde extérieur. Poser des conditions au paysage, c'est le considérer non seulement comme un signifiant interprétable, mais, pour reprendre le terme utilisé par Berque lui-même, comme un prédicat ou une médiance (Berque, 2008), c'està-dire une forme sous laquelle le monde extérieur est saisi par les hommes - une peau que revêt ce monde, ici, mais pas nécessairement ailleurs, à une époque, mais pas à un autre. Le paysage, comme identification spécifique, correspond donc à un état parmi d'autres possibles de la relation écouménale ${ }^{9}$. P. Descola parle quant à lui d' "ontologies» — totémique, analogique, animique et naturaliste - le paysage étant reconnu comme consubstantiel à cette dernière ; mais l'idée générale est similaire.

\footnotetext{
9 . «Je professe [...] l'idée que le paysage est non seulement historique, mais écouménal, c'està-dire qu'il dépend de la manière dont s'établit, sur terre, le milieu existentiel propre à telle ou telle société. Le paysage n'est donc pas universel ; il est contingent comme l'histoire et concret comme l'écoumène (la relation de l'humanité à l'étendue terrestre).» (A. BERQUE. 2008, p. 45).
} 
Ces deux théories suffiraient à elles seules à montrer que poser le problèmes de l'« invention » du paysage — aborder celui-ci comme une réalité contingente qui n'émerge qu'à certaines conditions - ne revient pas nécessairement à s'inscrire dans une perspective constructiviste. A. Berque refuse au reste d'employer le terme «invention» précisément parce qu'il s'est trop souvent trouvé affilié à l'idée de «construction sociale de la réalité » (Berque, 2008). Mettant en avant l'occidentalité et la modernité du paysage, Berque et Descola insistent sur son rôle constituant relativement à une identité culturelle ontologiquement fondée, plutôt que sur la propagation acculturante, hors de l'Occident, de cette forme de relation à l'environnement. C'est là la conséquence d'un refus de détacher ce regard du socle ontologique auquel on le pense rivé. Donner au paysage le rôle d'une vision du mode ontologiquement associée à la modernité et à l'Occident n'invite pas non plus, et pour la même raison, à explorer l'hypothèse de son implication dans les rapports de pouvoir existant au sein même de cette société moderne et occidentale, supposée cosmologiquement homogène.

\section{LES GRANDS RÉCITS ANGLO-AMÉRICAINS}

Or, de ce double point de vue, le paysage qui ressort des récits de fondation proposés par Berque et Descola se distingue de façon très nette de celui auquel donnent corps les récits proposés Outre-Manche et Outre-Atlantique à partir du début des années 1980. Le point de partage central entre ces deux grandes familles théoriques semble se situer, précisément, au niveau de la manière dont elles envisagent cette distanciation qui, depuis deux siècles et pour de nombreux auteurs, constitue la première des conditions de possibilité du paysage.

\section{Paysage et naissance du capitalisme : le grand récit de Cosgrove}

Que les courants français et anglo-américain de la pensée paysagère soient demeurés longtemps largement étrangers l'un à l'autre est ce que tend en particulier à montrer le peu d'écho qu'ont eu, en France, au moins jusqu'à ces toutes dernières années, les œuvres majeures associées au mouvement des Cultural landscape studies. On pense en particulier au Social formation and symbolic landscape du géographe anglais Denis Cosgrove (1984), pourtant considéré non seulement outre-Manche, mais aussi dans de très nombreux pays du monde, comme une référence majeure en ce domaine du paysage ${ }^{10} ;$ l'absence de traduction en français de l'ouvrage devant être plutôt considéré comme un symptôme que comme une explication de ce phénomène. On peut faire à peu près le même constat à propos de l'œuvre majeure de J.B. Jackson, Discovering the vernacular landscape, parue la même année. Même si ce dernier ouvrage a fait l'objet en 2003, d'une traduction en français, ces deux piliers des études britannico-américaines sur les «paysages culturels» n'ont pas influencé

\footnotetext{
10. J'utilise - et je citerai ci-dessous - l'édition italienne : Denis COSGROVE, Realtà sociali e paesaggio simbolico, Milan : Ed. Unicopli, 1990. Les autres travaux de D. Cosgrove n'ont pas eu non plus les honneurs de la traduction.
} 
directement la littérature paysagère produite en France dans les années 1980$2000^{11}$.

C'est dans l'ouvrage de Cosgrove et dans un article publié l'année suivante dans les Transactions of the Institute of British Geographers sur " Prospect, perspective and the evolution of the landscape idea » (Cosgrove, 1985), que réside pour l'essentiel la thèse formulée par ce géographe sur les « origines » du paysage. Cette thèse sera par la suite prolongée et amendée par Cosgrove, dans un sens sur lequel je reviendrai.

D'inspiration marxiste, le récit cosgrovien est axé sur le lien établi entre émergence d'une vision et d'une représentation paysagère du monde environnant, fin du féodalisme, développement du capitalisme marchand et ascension d'une classe sociale qui se représente à elle-même et donne à voir sous les traits d'un paysage l'espace sur lequel s'étend sa domination ${ }^{12}$. Cosgrove invite ainsi à regarder le paysage comme cette nouvelle forme de regard adapté à la manière propre à une classe émergeante de concevoir la possession du sol et l'usage des ressources.

La Renaissance italienne est le moment et le lieu où commence à se dessiner, selon Cosgrove, ce rapport paysager au monde. Le géographe anglais a ainsi montré un intérêt particulier pour la Vénétie du XVIème siècle, à laquelle il a consacré son The Palladian Landscape (1993). Cette région illustre de manière particulièrement spectaculaire, à travers le grand mouvement de réinvestissement dans les biens immobiliers de la Terre Ferme des capitaux d'origine commerciale accumulés par l'aristocratie vénitienne, le processus socio-économique auquel Cosgrove attribue l'émergence de ce qu'il appelle volontiers l'«idée » de paysage. Celle-ci fait irruption, selon lui, quand la valeur de la terre se transforme, en passant de la valeur d'usage, qui est celle que la terre revêt dans le système féodal, à la valeur marchande (ou la valeur d'échange), que le capitalisme lui confère. Le paysage émerge au moment où s'impose cette aliénation de la terre son objectivation comme "pure » propriété indépendante de sa valeur d'usage et en réponse aux résistances que rencontre le nouveau rapport à la ressource et à l'espace qui s'instaure alors. Pour Cosgrove, le paysage a en effet constitué le moyen sinon de masquer cette mutation, au moins de la faire accepter :

«L'idée de paysage en Occident et son expression artistique ont servi en partie à promouvoir idéologiquement l'acceptation du rapport de propriété, en soutenant au moment où il s'impose l'image d'un rapport non aliéné, de la terre en tant que valeur d'usage. » (Cosgrove, 1984, p. 75)

L'idée de «paysage » appartient ainsi à la transition entre deux systèmes socio-économiques et culturels. Elle émerge et triomphe durant la période — qui

11 . Des traductions et une analyse de certains textes de Jackson sont parus dans Le Visiteur, $\mathrm{n}^{\circ}$ 5, 2000. Deux ouvrages du même auteur (Jackson 2003 et 2005) ont été traduits en français. Sur la pensée de Jackson, voir : BESSE, 2000 et 2003.

12. "Landscape first emerged as a term, an idea, or better still, a way of seeing the extemal world, in the fifteenth and early sixteenth centuries. It was, and it remains, a visual term, one that arose initially out of renaissance humanism and its particular concepts and constructs of space. Equally, landscape was, over much of its history, closely bound up with the practical appropriation of space. » (COSGROVE. 1985, p. 46). 
correspond pour Cosgrove à l'époque moderne des historiens - où la mutation de la valeur de la terre fait face à de nombreuses résistances. La force culturelle de cette idée n'est ainsi vraiment importante que jusqu'au moment de «la stabilisation de l'hégémonie du capitalisme urbain industriel et de la culture bourgeoise de la propriété ». Le paysage perd dès lors

«sa force artistique et morale et devient un résidu de la production culturelle, considéré ou comme un élément de subjectivité purement individuelle, ou comme un objet d'étude académique scientifiquement définie, en particulier en géographie » (Ibid.)

Avant ce moment, l'idée de paysage a pleinement intégré la tension entre deux modes de rapport à la terre, qui correspondent aussi pour Cosgrove, à deux postures de l'homme par rapport au monde perceptible qui l'environne et à la nature :

«Dans une économie naturelle [i.e. féodale], le rapport dominant entre les êtres humains et la terre est celui de l'insider, un rapport non aliéné fondé sur la valeur d'usage et l'interprétation [de la nature] en un sens analogique. Dans une économie capitaliste nous nous trouvons face à un rapport entre un possesseur et une marchandise, un rapport aliéné dans lequel l'homme a le rôle de l'outsider et interprète la nature dans un sens causal.

[...] L'idée de paysage maintient ensemble [ces deux] types de rapport en une unité instable, menaçant toujours de tomber dans le subjectivisme irréfléchi de l'insider, pour qui le sentiment pour la terre est incommunicable avec les langages codés de l'art, ou dans l'objectivation de la terre comme propriété pure et simple, c'est-à-dire dans le point de vue de l'outsider, pour qui l'aliénation est complète... » (Ibid.)

Tout en intégrant ces tensions et en assumant plus ou moins chaotiquement cet aller-retour entre points de vue de l'insider et de l'outsider, l'idée de paysage a pour condition de possibilité l'émergence de cette vision objectiviste de la nature et de l'espace géographique qui est consubstantielle au capitalisme. Avec l'économie capitaliste s'imposent de nouveaux modes de représentation au service de la mesure du monde et qui sont aussi ceux-là même qui ordonnent sa représentation artistique. Cosgrove accorde, avant d'autres, une grande importance à ce qu'il appelle l' « idéologie visuelle de la perspective linéaire » qui fit de l'univers des images celui où s'exprima en priorité ce nouveau mode de rapport à la nature et à la terre. Mais si l'émergence du paysage suppose la possibilité d'une posture spectatoriale et distanciée de l'homme vis-à-vis du monde environnant, le point de vue paysager n'en est pas pour autant assimilable, dans l'esprit de Cosgrove, au point de vue esthétisant et subjectif. La subjectivité est plutôt le propre de l'insider, c'est-à-dire de celui qui n'objective pas la valeur de son environnement. Chez Cosgrove, il n'y a pas, d'autre part, d'assimilation entre point de vue esthétique et point de vue artistique. L'artiste, et notamment le peintre, au moins à la Renaissance, apparaît surtout complice - et Berque rencontrera Cosgrove sur ce point — de ce point de vue distancié et objectivant sur la nature qui est aussi celui du savant.

$\mathrm{Si}$, donc, un lien est établi par Cosgrove entre le paysage et la vision distanciée, l'avènement de cette dernière est précisément ce qui va inciter à la récupération d'une forme de rapport à l'espace et à l'environnement antérieure au 
rapport à la réalité qui émerge avec cette distanciation. Le rapport paysager au monde est ainsi celui par lequel le point de vue de l'insider lui-même entre — via la peinture ou l'aménagement de l'espace in situ — en représentation, ou, en d'autres termes, devient le référent même de la construction d'une image idéale des territoires soumis à l'exploitation capitaliste.

La position de Cosgrove peut être de ce point de vue rapprochée de celle de J.B. Jackson, même si ce dernier s'est plus écarté que lui d'une théorie de l'émergence du regard et du sentiment paysager. Pour Jackson lui-même, la relation paysagère au monde est à la fois solidaire d'un partage qui s'opère et d'un lien qui se noue entre un " monde intérieur " et un "monde extérieur " mis à distance. Comme forme de regard, le paysage ne serait ainsi pas seulement un filtre à travers lequel passerait l'appréhension du monde environnant, mais la voie par laquelle ce dernier acquiert une extériorité — devient, précisément, un « environnement » - et se voit dans le même mouvement construit comme objet d'appréciation et support d'une mise en scène du cadre de vie.

Dans un texte publié en 1979 dans le dernier numéro de la revue dont il est le fondateur, Landscape, Jackson rapproche, filant une analogie qui fera florès, paysage et théâtre ${ }^{13}$. S'il ne parle pas d'une «invention» ni d'une " origine » renaissante du paysage, reconnaissant au Moyen Âge ce qu'on pourrait appeler sa propre paysagéité, il constate que le théâtre tel que nous le connaissons aujourd'hui, qui fait son apparition en Italie à la fin de la Renaissance, donne forme à une relation de l'homme à la nature faisant de cette dernière un "monde environnant », impliqué dans un spectacle dont l'homme est à la fois le spectateur et l'acteur. Le théâtre et le paysage ne sont ainsi pas seulement complices parce que le premier fait participer le second, comme décor, à la scénographie, mais parce que tout deux sont solidaires de ce nouveau dispositif visuel et spectatorial à travers lequel l'homme perçoit, désormais, l'univers et la place qu'il occupe en lui. Jackson ajoute toutefois cette remarque importante :

«Il est caractéristique de cette époque qu'elle définisse le paysage d'une autre façon que nous : l'expression désignait à la fois l'arrière-plan d'un tableau et un décor de scène, cet élément qui donne forme à la composition et la situe, mais qui n'appartient pas vraiment à l'argument. » (Jackson, 2005, p. 115)

On a là une relativisation de l'innovation apportée par la Renaissance, moment essentiel dans l'esprit de Jackson, mais qui n'est toutefois qu'un moment dans une histoire des modes paysagers de perception dont il s'emploie ailleurs luimême à proposer une périodisation, dans laquelle on peut voir l'une des sources d'inspiration du récit de Cosgrove. Le scénario historique proposé par Jackson est fondé sur l'opposition, centrale dans la pensée de cet auteur, entre "paysage politique » et « paysage vernaculaire ». Le paysage politique reflète pour Jackson la volonté d'un pouvoir centralisé organisant l'espace à partir des normes qu'il produit, des idéaux qu'il porte et des messages qu'il cherche à délivrer. Ce

13 . JACKSON, [1979] 2005. Cette analogie est également développée par Eugenio TURRI (1998) ; et K. OLWIG, comme nous allons le voir, mettra en avant l'importance du théâtre dans le processus par lequel le pouvoir central, dans l'Angleterre absolutiste de la première moitié du XVIIème siècle, s'empare de la notion de paysage pour en transformer profondément la signification. 
paysage entretient une relation de transcendance avec le substrat biophysique existant et il représente ainsi, par excellence, le paysage moderne. Le paysage vernaculaire - que Jackson appelle aussi « paysage habité » ou «paysage vécu » - est au contraire le produit spontané de la pratique sociale, qui compose avec l'existant. Jackson distingue ainsi un temps du "paysage vernaculaire", correspondant à une époque médiévale qui n'est aucunement étrangère au sentiment du paysage. A cette période succède celle d'un paysage conditionné par la modernité politique, lui imposant les abstractions géométriques qu'elle a conduit à concevoir; puis vient l'époque du «nouveau vernaculaire». On retrouve bien ici, dans cette alternance de moments paysagers dans la longue durée, cette dialectique du vernaculaire et du distancié, qui est au cœur de la pensée de Cosgrove ${ }^{14}$. Cette position relativiste, plus historienne qu' " originiste », n'est toutefois pas celle qui a dominé la littérature paysagère de ces dernières décennies.

\section{Les fondements d'une critique socio-politique du paysage}

Ce qui frappe dans les thèses d'un Cosgrove, vu en particulier de France, est évidemment la place donnée à une approche socio-politique du paysagement du rapport des Occidentaux au monde. Le géographe anglais a de ce point de vue ancré dans un récit et une thèse "originiste » des idées défendues avant lui, notamment, par Raymond Williams, auquel revient d'avoir fait du paysage l'une des problématiques clés des Cultural studies. L'auteur de The country and the city (1973) avait ainsi lui-même montré que l'histoire des « significations culturelles » du paysage ne s'éclairait que dans son rapport à l'évolution des modes d'exploitation des territoires, des techniques et des structures foncières (Williams, 1977). Au-delà, Williams et Cosgrove regardent tout deux le paysage comme un mode spécifique d'appréhension du réel dont la codification a joué à certaines époques un rôle important dans l'économie des rapports sociaux et dans la représentation/légitimation du pouvoir, notamment en offrant la possibilité d'une « naturalisation » des inégalités et des relations de domination. Cette perspective a été prolongée par les cultural landscape studies sur divers terrains et en particulier, ces dernières années, sur celui des rapports entre colonisation, impérialisme et paysage; et l'on désigne aujourd'hui couramment par l'expression "imperial landscape», emprunté à un retentissant essai du géographe américain W.J.T. Mitchell (1994b), ce paysage appréhendé comme une idéologie visuelle au service du pouvoir.

Ce qui sans nul doute explique largement l'importance prise, à l'origine du mouvement des Cultural landscape studies, par cette approche socio-politique de la question paysagère est l'existence de cet arrière-plan historique très prégnant que constitue, dans l'histoire socio-culturelle du monde anglo-américain, l'émergence et le rayonnement, à partir du XVIIIème siècle, d'une culture du «pittoresque » indissociable de l'image que construisent d'elles-mêmes les nouvelles élites de ce temps, actrices et principales bénéficiaires de l'essor

\footnotetext{
14. Sur la scansion Moyen Age vernaculaire, modernité politique, nouveau vernaculaire, dans Discovering the vernacular landscape, cf. J.M BESSE. 2009, Chap. III « Le paysage, entre le politique et le vernaculaire $»$.
} 
agricole et industriel. Cette culture par essence « paysagère » s'exprime, on le sait, de façon privilégiée à la fois dans l'art du landscape gardening, indissociable luimême de nouvelles approches de la peinture, ainsi que dans les pratiques du voyage et de la promenade, qui mettent en jeu un art, élitiste et savamment codifié, d'éprouver les paysages. Williams et Cosgrove (1984) ont participé eux mêmes, en retrouvant sur ce terrain nombre d'historiens britanniques - John Barrell (1980), Ann Bermingham (1989), Copley et Garside (dir., 1994), Tom Williamson (1995), Stephen Daniels (1999)... — à construire une nouvelle lecture de cet épisode de l'histoire de l'art et des sensibilités, en l'abordant sous l'angle d'une analyse des rapports entre cette culture datée du paysage et les mutations contemporaines de la société britannique ${ }^{15}$. Ils ont ainsi participé à montrer comment la question paysagère peut être appréhendée, au XVIIIème siècle et au début du siècle suivant, comme le terrain symbolique sur lequel s'expriment les conflits inhérents à une mutation profonde des rapports de domination, et comment un nouvel ordre social se fabrique, dans le miroir du paysage, des images idéales de lui-même - ou comme l'écrit Cosgrove lui-même, comment « le pouvoir visuel donné par le mode paysager de vision [the landscape way of seeing] prolonge le pouvoir exercé par les hommes sur l'espace possédé » (Cosgrove, 1985, p. 1).

\section{Du paysage-communauté au paysage-spectacle : les thèses d'Olwig}

C'est au milieu des années 1990 qu'apparaît un récit porteur d'une interprétation vraiment nouvelle des origines du paysage et du sens qu'il convient de donner à la notion. Il est l'œuvre du géographe américain — et philologue d'origine - Kenneth Olwig, qui est parti de l'examen d'un corpus de sources de la fin du Moyen Âge et du début de l'époque moderne concernant l'Europe du nord Olwig (1996, 2003) observe que les mots Landschaft, landskab, landscap désignent alors dans les textes des communautés largement autonomes vis-à-vis des pouvoirs centraux, correspondant à des territoires gouvernés en référence à un droit coutumier qui intègre la spécificité des configurations du milieu physique local et garantit la justice dans le partage des ressources. Les mots que l'on peut traduire par «paysage » désignent alors également ce droit lui-même, mais aussi les représentants des Landschaften au sein des assemblées. Olwig démontre ainsi que ce qu'il appelle la « nature substantielle du paysage » n'est pas à chercher, au moins pour ces régions, dans un processus d'esthétisation du pays perceptible, ou dans sa mise en spectacle, mais qu'elle a trait aux notions de communauté et de justice, et qu'elle renvoie en priorité aux pratiques sociales et environnementales — au territoire vécu, habité et exploité. Il va jusqu'à avancer que les peintres flamands du XVème siècle eux-mêmes, en qui beaucoup ont vu les traducteurs inspirés d'une esthétisation du rapport au monde environnant, auraient d'abord représenté dans leurs œuvres ce paysage-communauté lui-même, au moment où la forme politique qu'il incarnait était menacée par les grands états monarchiques.

\footnotetext{
15. Cf. sur cette question notamment et, plus largement, sur les recherche anglo-américaines concernant les « paysages culturels » l'analyse bibliographique de Dianne HARRIS, 1999.
} 
Olwig situe dans l'Angleterre absolutiste de la première moitié du XVIIème siècle le moment du basculement du sens de Landschaft et de landscape. On assiste alors selon lui à une véritable captation et à un détournement du concept par la monarchie, qui commence à associer sa propre image à des vues paysagères, présentées notamment dans le théâtre des masques, à travers lequel la monarchie se met elle-même en scène. Le landscape devient alors le spectacle du pays "amélioré », c'est-à-dire un territoire dont le roi fait fructifier les ressources et qu'il embellit. Il prend une valeur visuelle et spectatoriale, en devenant la représentation d'un pouvoir. On retrouvera au siècle suivant cette notion d'improvement au cour de la culture du pittoresque et, spécifiquement, de la pratique du landscape gardening, par laquelle une élite sociale montre sa capacité à tirer vers la belle nature le donné brut livré aux sens de l'observateur.

Le grand récit paysager d'Olwig bouscule donc les certitudes que l'on affichait naguère sur les origines renaissantes du paysage et sur le lien entre vision paysagère et modernité. Il condamne sans possibilités d'appel les scénarios de type rogiériste, associant l'histoire du paysage à une sorte d'odyssée du sentiment esthétique. Il porte aussi une critique radicale de la théorie d'Augustin Berque, pour qui l'existence du mot «paysage » est la condition absolue permettant de conclure à l'existence d'une relation paysagère aux milieux. Olwig montre le caractère changeant du sens des mots, mais aussi et surtout que le paysage ne peut être toujours et partout associé à une relation esthétique au monde environnant.

In fine, le récit d'Olwig rejoint et valide néanmoins l'accent depuis longtemps mis par les géographes anglo-saxons sur l'instrumentalisation politique du paysage et sur le lien qui unit «naturalisation » du pouvoir et mise en spectacle et en représentation du monde environnant. Il prolonge et valide aussi la piste qu'avait ouverte dès le début des années 1980 J.B. Jackson, en proposant la distinction entre «paysage politique » et "paysage vernaculaire » (Jackson, 2003). C'est ce dernier paysage, plus vécu que regardé, plus habité que composé, que le grand récit d'Olwig participe à faire remonter à la surface.

Ce paysage-là, en quelque sorte consacré par l'enquête philologique et historique d'Olwig, a néanmoins toujours été présent dans les théories angloaméricaines, comme en creux, à travers par exemple la tension que Cosgrove voit s'installer au cœur de la notion de paysage entre des spatialité inhérentes au féodalisme et au capitalisme. Et Jackson lui-même, dès le début des années 1980, remarquait, quoique sans développer le constat, qu'il y a un millénaire, les mots Landschaft et Landscape «n'avaient rien à voir avec la mise en scène et l'évocation du théâtre » (Besse, 2012, p. 86). Le grand récit d'Olwig donne ainsi la caution de l'histoire et d'un grand récit des origines à une idée du paysage qui met en jeu l'opposition de deux rapports opposés de l'homme au monde environnant. Il ouvre aussi la voie à l'enrichissement du sens de cette opposition, en l'associant potentiellement à des arrière-plans nouveaux, dans lesquels se font face l'individu et la communauté, mais aussi rapport de production et de consommation de l'homme au territoire (Cosgrove, 2006). Contrairement à Cosgrove, qui voit cette opposition comme portée par la notion de paysage ellemême, Olwig l'inscrit, reprenant ce que Jackson avait esquissé, dans une trajectoire historique de la notion. 


\section{LES GRANDS RÉCITS PAYSAGERS COMME MARQUEURS ÉPISTÉMIQUES}

Cette rapide traversée de quelques-uns des grands récits paysagers produits durant les deux derniers siècles ne visait pas à en dresser le recensement exhaustif. Seuls ont été retenus ceux qui ont paru à l'auteur de ces lignes les plus représentatifs de leur époque et ceux qui exercent aujourd'hui, ou ont exercé dans le passé, la plus forte influence. Reste, sur ces bases désormais posées, à interroger la signification profonde de la production de ces récits, de leur émergence au début du XIXè siècle et de leur réémergence récente, en tirant au maximum parti de ce regard panoramique qui embrasse la longue durée. En d'autres termes, il s'agit maintenant de tenter de débusquer ce qui est sous-jacent à cette interrogation sur les origines du paysage, les enjeux non exprimés qu'elle porte et, au-delà de la diversité des réponses apportées par les différents auteurs, les déterminations historiques poussant à un questionnement qui leur est, par delà les siècles, commun.

\section{Des origines à la généalogie : déplacer la question}

Un constat d'abord s'impose : le propos des grands récits paysagers n'est pas à proprement parler d'ordre historique. L'histoire disparait ici derrière les commencements. Ces récits abordent ainsi le paysage comme relation au monde extérieur une fois pour toute instituée et déterminée par les conditions qui président à son avènement même. Ce moment originel, où le paysage advient, n'installe donc qu'en apparence une discontinuité sur l'axe du temps. Il est en réalité le moyen de dire, à l'inverse, une continuité historique, dans laquelle pourra s'abriter et subsister le paysage que l'on s'efforce de construire et d'habiliter. Car telle apparaît d'abord la fonction des énoncés théoriques dans lesquels s'inscrivent les grands récits : construire, habiliter et légitimer dans les configurations qu'on lui confèrent l'objet paysage, en lui assignant une définition, des usages, usagers et experts légitimes, un statut scientifique, artistique et politique déterminé. On peut ainsi poser que la spécificité la plus fondamentale de cet objet paysage réside ainsi peut-être en ceci que sa construction apparaît indissociable d'un discours des origines et du constat d'une relativité historique. L'objet paysage, en d'autres termes, apparait soumis depuis les alentours de 1800 à un régime d'objectivation qui inféode son existence à l'invention d'une invention.

L'interprétation proposée ici des grands récits du paysage a pour fondement un déplacement de la question qu'ils posent. Il s'agit de passer d'une problématisation, d'ordre originiste, de l' «invention», comprise comme émergence de la chose (ici du paysage comme réalité), à une problématisation, d'ordre généalogique (au sens de Foucault), de l'objectivation entendue comme construction datée de l'objet. Ces récits peuvent ainsi être regardés comme les témoins d'un processus d'objectivation du paysage, auquel ils apportent euxmêmes une contribution importante. 
Il ne s'agira donc pas de discuter, ici, les théories proposées par les auteurs cités plus haut. Celles-ci ne nous intéressent qu'en tant qu'elles nous parlent des déterminations historiques conduisant à une construction ou une reconstruction $d u$ paysage comme objet. Les grands récits du paysage apparaissent, dès lors qu'on les appréhende sous cet angle, comme les archives de tournants épistémiques dont ils reflètent au moins certains aspects. Tenter de dire lesquels est l'objet de ces considérations conclusives.

\section{Aux fondements de l'objectivation du paysage}

On peut penser que l'apparition au début du XIXè siècle d'un questionnement sur les origines du paysage marque l'étape décisive du premier véritable processus d'objectivation du paysage en Occident. Des débats du Siècle des Lumières sur le paysage comme genre pictural et jardinier n'émerge qu'une objectivation partielle, de portée limitée. Dans ce qui s'offre à la perception directe — non médiatisée par les œuvres - le paysage n'est jamais appréhendé au XVIIIè siècle que comme une collection désordonnée d'objets, réservoir de motifs pour le peintre ou le créateur de jardins, d'échantillons pour le naturaliste, de caractères pour le physiognomoniste, etc. (Briffaud, 2013). Les voisinages, dans l'étendue perceptible, et donc le paysage en tant qu'agencement d'éléments dans un espace, ne signifient pas. La nature n'est pas présente en eux. Le regard du naturaliste et de l'artiste, ne rencontrent, en se tournant vers le monde extérieur, qu'une nature surchargée d'ajouts, aux composants déplacées par les révolutions du globe et aux formes rhabillées par ce temps qui passe ${ }^{16}$. Briser la gangue, remettre en place ce que l'histoire a emporté, nettoyer êtres et choses des dépôts laissés par elle à leur surface ; rendre, en un mot, à l'évidence perceptible leur vérité substantielle (i.e. leur caractère distinctif), tel est l'objectif que naturalistes et peintres poursuivent dans leurs cabinets et leurs ateliers, à l'abri des sensations et des idées confuses que peuvent faire naître les paysages du dehors. C'est, précisément, la non signification de ces derniers qui participe à conférer à l'art et à la science naturelle leur fonction première.

Mais réordonner la nature et lui redonner une visibilité ne passe pas, chez le naturaliste et chez l'artiste, par une même opération. Le savant, dans son cabinet, soumet les éléments livrés à la perception directe à l'ordre que prescrit la grille des taxinomies, sur laquelle repose l'identification du même et du différent. Le peintre et le jardinier, dont l'art relève de la composition, mettent quant à eux en relation ce que le naturaliste de son côté juxtapose et distingue. Ils recréent, sur la toile ou dans le jardin, un tout-ensemble dans lequel nombre de théoriciens commencent à voir, dès l'aube des Lumières, la source d'une beauté typiquement picturale ${ }^{17}$. Dans la notion de pittoresque émergeant dès cette période, mais qui

16. «... l'expérience, écrit à ce propos Foucault, ne nous livre pas, tel quel, le continu de la nature. Elle le donne à la fois déchiqueté [...] et brouillé, puisque l'espace réel, géographique et terrestre, où nous nous trouvons, nous montre les êtres enchevêtrés les uns avec les autres, dans un ordre qui, par rapport à la grande nappe des taxinomies, n'est rien de plus que hasard, désordre ou perturbation. » (Foucault, 1966, p. 160-161).

17. «Le Tout ensemble est un résultat des parties qui composent le tableau, en sorte néanmoins que ce tout qui est une liaison de plusieurs objets ne soit point comme un nombre composé de 
n'est vraiment théorisée qu'à la fin du XVIIIè siècle, il y a celle d'une possible substitution de l'œuvre peinte à la réalité — d'un paysage composé à un paysage-collection dont l'œil artialisé du connaisseur serait capable d'opérer la transmutation en tableau. C'est ainsi à l'art et à lui seul qu'appartient vraiment, au XVIIIè siècle, l'objet paysage.

Pour que la science vienne rejoindre l'art sur ce terrain du paysage, il a fallu que ce dernier puisse commencer à apparaître comme une composition signifiante dans la nature perceptible elle-même, et non plus seulement dans la peinture ou le jardin, et par leur seule médiation. Cette appropriation a eu paradoxalement pour condition une invisibilisation du naturel ${ }^{18}$. Le paysage commence autrement dit à signifier, en tant qu'agencement d'éléments naturels quand, au tournant des $\mathrm{XVIII}^{\circ}$ et $\mathrm{XIX}^{\circ}$ siècles, la nature elle-même se déplace du caractère perceptible vers l'organisme et son fonctionnement; quand elle devient force de vie, énergie créatrice, natura naturans et que le temps, cessant de n'affecter que l'espace extérieur aux êtres et aux choses commence à travailler en leur sein même. Le paysage, qui n'était qu'un contexte, peut dès lors apparaître comme l'expression, dans le champ du perceptible, de ce mouvement dans lequel l'essence même des choses est emportée. Il devient la représentation d'un processus dont on ne perçoit, en surface, que les symptômes. La réalité de la nature recule alors, dans le champ visuel, en une représentation partielle d'ellemême. Il ne s'agit plus de décaper, pour atteindre la substance, mais d'extrapoler, pour compenser l'incomplétude de ce qui offre à être vu. Alors, et alors seulement, quand le temps s'est emparé de la chose même et que le spectacle des dehors de la nature n'offre plus que la trace d'un avant et le signe d'un après, le paysage commence à signifier comme agencement et comme situation. Quand l'histoire ne masque plus la substance des êtres et des choses, mais devient la grande ordonnatrice des "connexités » (A. de Humboldt) entre les éléments naturels, les voisinages cessent en effet de n'être que hasard et l'espace, tel qu'il s'organise dans le champ visuel, commence à contenir et à révéler ce qui ne se voit pas directement: un passé et un devenir. Une lecture des agencements est désormais possible.

\section{Les grands récits romantiques du paysage et la figure de l'observateur}

L'émergence des grands récits du paysage occidental au début du XIXè siècle prend sens dans ce contexte d'une transformation des relations du naturel à l'environnement perceptible, c'est-à-dire du statut du visible et du regard luimême. Elle s'éclaire en d'autres termes par le passage, que ces récits marquent et illustrent, d'un régime scopique à un autre. Cette expression de régime scopique est ici employée dans le sens que lui donne Jonathan Crary, pour qui elle désigne

plusieurs unités indépendantes et égales entre elles, mais qu'il ressemble à un tout politique, où les grands ont besoin des petits, comme les petits ont besoin des grands [...]. Car l'effet qui en résulte consiste dans une subordination générale [...] où le mérite de chaque chose n'est fondé que sur une mutuelle dépendance. Ainsi pour définir le Tout ensemble, on peut dire que c'est une subordination générale des objets les uns aux autres, qui les fait concourir tous ensemble à n'en faire qu'un. » (De Piles, [1708] 1989, p. 69).

18 . Ici encore, je renvoie à M. FOUCAULT, 1966, chap. V. 
le statut daté accordé à la fois au regard et au visible dans leur rapport au " réel » et à la beauté - c'est-à-dire une certaine façon de se représenter l'efficience de l'œil et la valeur de ce qu'il lui est donné d'embrasser. Tout régime scopique recèle ainsi une co-définition implicite, ou une co-institution, du sujet observateur et de la « réalité » observée.

Qu'une même façon de se représenter la nature de la vision et du visible rassemble, au temps de Schlegel et de Humboldt, différents domaines de la connaissance est ce que suggère un détour par la conception romantique du savoir historique. François Hartog montre ainsi comment le projet de l'Histoire de France de Michelet apparaît tout entier fondé sur un renoncement à la posture du regardeur-historien, se contentant de mettre en récit ce qui tout naturellement tombe sous son regard, au profit de l'affirmation d'une « vision » qui ausculte les tréfonds du corps social et politique, à la recherche d'une vérité que la révolution a ramené à la lumière, mais qui est retournée, depuis, dans les ténèbres d'où elle était un bref instant sortie. " D'une évidence octroyée à une évidence retrouvée, traduite et transmise, tel est le contrat [de l'historien] » (Hartog, 2005, p. 162).

Cette dernière formule décrivant la trajectoire de l'histoire et de l'historien pourrait parfaitement être utilisée pour décrire la trajectoire du regard et du sentiment que décrit Humboldt, c'est-à-dire à la fois la sienne propre et celle qu'il projette sur l'humanité européenne. Le cosmos humboldtien est lui aussi placé sous le signe - pour reprendre une expression que Hartog emprunte lui-même à Foucault - de l'« invisible visibilité ». L'évidence octroyée, chez Michelet contenue dans le spectacle de la révolution, correspond chez Humboldt à cette révélation qui réside en l'instant (à l'échelle d'un vie) ou l'époque (à l'échelle de l'histoire) de la découverte de la montagne tropicale, l'auteur de Cosmos transférant sur les colons du XVIè siècle le sentiment, par lui-même éprouvé au pied de la cordillère, d'atteindre à une vérité de la nature qui cesse, ici, de n'être qu'abstraite, en s'incarnant dans l'évidence du visible et l'immédiateté d'une sensation. Et le contrat que Humboldt propose aux sciences naturelles est analogue à celui qui lie l'historien : retrouver, traduire et transmettre, en la faisant pénétrer à nouveau dans les paysages perceptibles, cette évidence un instant aperçue. La « vision » de Michelet, objectera-t-on, est d'ordre métaphorique, alors que Humboldt regarde la nature avec son œil physique. Mais l'essentiel est que tout deux fassent néanmoins référence à cette position un instant occupée, qui est celle du témoin direct, auquel il est donné de saisir d'un seul coup d'œil ce dont tout ce qui s'offrira par la suite à être vu ou entendu ne constituera que la trace ou le fragment, mais ne s'en mettra pas moins, en tant que tel, à signifier. Tel est la condition de possibilité que Humboldt assigne à la vision paysagère de la nature : être capable de faire entrer en chacun de ses spectacles le souvenir d'une illumination passée et de se servir de tous pour atteindre ce que peu d'entre eux peuvent directement accorder.

Hartog, s'appuyant sur Foucault (1963) ${ }^{19}$, montre au reste que le régime de visualisation qui caractérise l'histoire de Michelet est également semblable à celui qui se dégage, dans la médecine de cette même époque, de la codification des 19 . Notamment sur le chapitre «Voir, savoir» de FOUCAULT, Michel. 1963. Naissance de la
clinique. Paris : Presses Universitaires de France, p. 107 sq. 
pratiques de l'observation clinique, quand la clarté cesse d'être une propriété des corps perceptibles, pour devenir l'apanage de l'œil lui-même, du regard lent et attentif

«qui les parcourt, les contourne et peu à peu les pénètre en ne leur apportant jamais que sa propre clarté. Le séjour de la vérité dans le noyau sombre des choses est paradoxalement lié à ce pouvoir souverain du regard empirique qui met leur nuit à jour. » (Foucault, 1963, p. X)

C'est dans cette transformation profonde des conceptions du visible et de l'observation elle-même, qui concerne tout à la fois l'art et la science, qu'il faut aller chercher les fondements principaux de l'attention portée à la question paysagère dans la première moitié du XIXè siècle. À travers les discours sur l'émergence du paysage, on ne fait rien d'autre alors que reconstruire, sur les ruines du rapport à la vision et au visible hérité de l'époque classique, un paysage dans lequel pénètre le nouveau sens que prend l'acte même de voir et de regarder. Le paysage devient, dans ce mouvement, l'une des figures dans lesquelles s'incarne cette nature à la vérité obscurcie, qui a perdu son évidence et sa clarté, et qui, précisément parce qu'on reconnaît et assume la distance inéluctablement prise avec elle, s'offre à la compétence du regardeur expert, ramenant vers la surface perceptible des choses une lumière que seule l'exploration patiente et pénétrante des profondeurs permet de faire à nouveau briller. Ce paysage qui existe sur le mode de la trace ou de l'archive a ainsi acquis une profondeur historique, ou plus largement représentationnelle, au sens où la finalité de la représentation est de rendre présent ce qui ne l'est plus ou pas : ce qui est passé, mais aussi ce qui est ailleurs. Or, la reconnaissance de cette épaisseur documentaire du paysage apparaît indissociable, dans la première moitié du XIXè siècle, de la relativisation culturelle de cet objet-paysage, qui, à travers les spéculations sur son avènement passé ou à venir est déjà appréhendé comme une certaine manière de voir le monde, porteuse de la spécificité d'une époque et d'une humanité européenne qui a acquis, à travers lui, une compétence visuelle et sensible fondant sa spécificité et, potentiellement, sa supériorité.

Des grands récits du paysage occidental conçus à l'époque romantique se dégage, ainsi, au point de rencontre entre l'homme et son environnement, la figure nouvelle de l'observateur, sur laquelle repose depuis deux siècles la résolution du paradoxe de l'invisible visibilité de la nature. Cet observateur n'est pas un simple regardeur compétent, prenant en charge le décryptage et l'évaluation d'un déjà-là perceptible. Son rôle n'est pas seulement d'apprécier, en connaisseur, les paysages, de les lire et de débusquer en eux les traces de réalités et de fonctionnements cachés, ni d'en diagnostiquer, en expert, les éventuels dysfonctionnements, ni encore, en artiste, d' "artialiser» l'environnement perceptible. L'observateur est aussi et bien plus fondamentalement, en faisant tout cela, le grand ordonnateur des épiphanies paysagères. À lui revient de révéler les paysages et de les «inventer», c'est-à-dire de les rendre disponibles à l'appréhension sensible et/ou savante et de les faire exister là où on ne les avait pas encore reconnus, en reconduisant dans le champ du perceptible une nature qui s'en est écartée, perçant ainsi l'enveloppe opaque qu'est devenu le monde à portée d'expérience et faisant remonter vers cette surface une signification enfouie dans le cœur obscur des choses. C'est à cet observateur qu'il revient d'établir la 
cartographie symbolique des espaces, de fixer les limites du regardable, du beau et du signifiant, et d'établir ainsi, pour tous et en vertu de son expertise, les discontinuités fondamentales qui ordonnent le rapport sensible des individus et des sociétés à leur environnement.

En proposant de lire l'histoire du paysage sous l'angle d'un avènement et donc d'une rupture entre deux époques distinctes, les grands récits paysagers produits au seuil de l'époque contemporaine apportent leur caution à l'autorité dont commence alors à se parer cet observateur-inventeur, auquel revient d'actualiser là où ses pas le portent et son regard se pose, ce moment décisif où le paysage advient. Dans l'exaltation de cette figure, qui incarne en ses formes nouvelles le mérite et la supériorité sociale, réside, on peut le penser, l'enjeu majeur sous-jacent à ces premiers récits et à l'entreprise d'objectivation du paysage qu'ils prennent en charge.

\section{Les récits paysagers contemporains et la crise de l'observation}

Sans doute est-il symétriquement possible de voir dans la réémergence récente des grands récits de l'émergence du paysage une expression de la crise de cette figure et de ses répercussions sur les institutions qui fonctionnent autour d'elle et par elle : savoirs constitués, politiques (d'aménagement des espaces et des territoires, de protection des beautés naturelles...), pratiques (le tourisme notamment, considéré en ses formes les plus profondément ancrées dans l'histoire).

L'importante contribution de la géographie à la réémergence contemporaines de la problématique des origines du paysage apparaît ainsi inséparable d'une critique fondamentale de la posture du géographe-observateur, dont l'objet serait immanent à un "donné perceptible » et dont les méthodes auraient l'expertise visuelle pour premier fondement. Les récits paysagers des géographes apportent en cela leur concours à la démolition méthodique, mise en œuvre surtout à partir des années 1950, d'un paysage géographique hérité (Tissier, 2003), mais ils proposent aussi un dépassement de ce point de vue en misant non pas sur un pur et simple abandon du paysage, mais - dans une perspective, en définitive, plus radicale - sur une reconstruction du concept, l'habilitant à reprendre place, sous sa forme rénové, au cœur de la discipline et ainsi à mener à bien, de l'intérieur, la tâche de subversion épistémologique qu'on lui assigne désormais.

Les théories de D. Cosgrove, notamment, reflètent bien cette double dimension, critique et réinstituante, du discours géographique sur l'origine du paysage. En mettant à jour les contenus idéologiques intrinsèques de la notion et du mode paysager de perception et de représentation du monde extérieur, elles questionnent la vision du géographe lui-même, l'invitant à cesser de penser son rapport au paysage sous l'angle de l'observation objective d'une réalité donnée. La déconstruction de la culture géographique du regard et de la représentation, exhumant ses héritages et ses idéologies sous-jacents, est posée par Cosgrove comme une tâche indispensable à une critique et une reconstruction épistémologique de la discipline (Cosgrove, 2007). Celle-ci passe, si l'on peut dire, par la généralisation d'une observation de l'observateur lui-même, dont le 
géographe n'est que l'une des incarnations historiques. Elle passe autrement dit par une quête étendue en de multiples directions des processus de construction culturelle de l'environnement perceptible, des codifications, des idéologies, des enjeux de pouvoir et de distinction sociale qu'ils portent; le paysage ne se présentant plus dès lors, selon la formule de Mitchell, « comme un objet à voir ou comme un texte à lire, mais comme un processus par lequel se forment des identités sociales et subjectives » (Mitchell, 1994a, p.1), au sens d'identités enracinées dans des codes de perception et des modèles esthétiques. Le centre de gravité de la discipline se déplace, dans le même mouvement, d'un environnement perceptible autodonnée vers sa construction même, qu'elle soit saisie à travers discours et représentations, ou à travers des structures matérielles. Le « paysage culturel », qui était pour Sauer (1925) et ses héritiers affaire de «morphologie » devient ainsi un écran sur lequel défile, devant le regard partial du spécialiste, les images d'un monde travaillé par l'idéologie.

La crise de la figure de l'observateur, qui ébranle en ses fondements mêmes une objectivation héritée du paysage, ne s'exprime pas seulement dans de telles reconstructions de l'objet. La crise, en ce domaine comme en tous les autres, réifie autant qu'elle disqualifie. Le scénario gombrichien, dont on a vu qu'il fonctionnait en véritable paradigme ordonnateur des discours contemporains sur les origines du paysage occidental, est ainsi support de la réactualisation d'un paysage placé sous la dépendance d'une médiation épiphanique. Celle-ci n'est toutefois plus associée, dans les récits que ce scénario venu de l'histoire de l'art inspire, à la figure héritée d'un observateur cumulant la capacité à lire le paysage comme trace d'un ordre naturel légal et à en révéler les beautés. Elle est tout entière confiée à un artiste libéré de la tâche de connaître et de faire connaître, ainsi qu'aux titulaires d'un savoir-éprouver acquis dans la familiarité avec les œuvres. Ce scénario paradigmatique n'en dérive pas moins directement de l'époque romantique. Il fait sien l'idée d'une émancipation progressive d'une représentation de « la nature », conquérant peu à peu les premiers plans de l'image (ou du poème), ou encore celle d'un dépassement inclusif de la narration par la description. Ce qui, à la Renaissance, commencerait à capter l'attention de l'observateur - ce dont il commencerait à pouvoir s'émouvoir, n'est rien d'autre, dans les récits contemporains, que cette nature schlégelienne, avec laquelle le regardeur a pris ses distances et vers laquelle il retourne avec nostalgie. C'est, en un mot, l'association de la vision paysagère du monde à un sentiment de la nature pré-construit et hérité, étroitement associée à une conception de la modernité, ellemême héritée du XIXè siècle, qui ordonne, dans l'ombre, une part importante des théories contemporaines sur les origines du paysage ${ }^{20}$; et c'est elle qui fonde la manière dont ces théoriciens se représentent a priori ce qui est ou ce qui fait, ou pas, paysage. Or, on ne peut sur cette base s'étonner, comme le note H. Brunon, qu'il soit impossible de retrouver, par exemple, dans la culture médiévale, un tel modèle paysager de perception et de représentation de la nature, tout droit venu du romantisme. Pour comprendre comment l'homme médiéval, ou un autre, perçoit son environnement, il faudrait commencer par ne pas le placer de force face à une

20. On peut suivre, sur ce point, Hervé Brunon, quand il affirme que « la thèse actuelle de la découverte du paysage à la Renaissance se nourrit en réalité d'une caractérisation de la modernité, en tant que nouveau rapport à la nature, héritée du XIX ${ }^{\circ}$ siècle. » (Brunon, 2006, p. 16). 
« nature » qui n'est pas la sienne et au sujet de laquelle il n'a, de fait, rien à déclarer.

L'histoire de l'art de ces dernières années a largement construit la critique de la vision du paysage portée par le scénario gombrichien (Brunon, 2006). Par delà la remise en cause de ce scénario lui-même, elle a fait apparaître les circulations nombreuses existant, en ce domaine du paysage, entre les arts et les sciences - circulations qui s'intensifient largement à l'époque romantique (Alpers, 1990; Klonk, 1997 ; Hoozee, 2007, etc.). À cet apport s'ajoute les travaux des historiens des représentations et des sensibilités, qui ont montré le rôle décisif joué par des sciences telles que géologie, la botanique, ou la géographie dans l' "empaysagement» de certains espaces (les littoraux et les montagnes notamment) (Corbin, 1988, Briffaud, 1994...). Les enseignements que l'on peut tirer d'une source telle que les grands récits paysagers du XIXè siècle accréditent eux-mêmes l'idée que la culture européenne ne se serait pas développée sur la base d'une disjonction radicale du sentir et du connaître, consubstantielle à ce que A. Berque appelle "le paradigme moderne-classique », mais plutôt au sein d'un art qui s'envisage lui-même (à l'époque classique et romantique) comme contribution à la connaissance et dans l'espace libéré par la discordance entre le principe d'un savoir scientifique fondé sur la distanciation objectivante et les économies complexes et plurielles du regard et de la sensibilité qu'engagent, en pratique, la construction d'une connaissance de la nature ${ }^{21}$.

Tout cela rend d'autant plus notable et significative la pérennité du scénario associant l'émergence du paysage à un procès d'épuration du regard et de la représentation, les autorisant à se saisir, une fois débarrassés de toute détermination religieuse ou cognitive, de la «simple présence des choses ». Pour comprendre cette pérennité, il faut sans doute la mettre en regard des ambitions épistémologiques que sert une telle idée du paysage. On peut en particulier penser que les récits gombrichiens participent d'une volonté plus ou moins consciemment assumée de ré-asseoir l'histoire de l'art et la philosophie esthétique à la fois sur la préséance reconnue à l'art dans la construction des sensibilités et sur l'affirmation d'une consubstantialité - dont le paysage se présente, en ses origines mêmes, comme la preuve ou l'incarnation — de la sensibilité à l'art, d'un côté, et à la nature de l'autre.

Mais il faut aussi considérer les enjeux sociétaux et politiques sous-jacents à ce type de discours, à l'heure d'une revalorisation/réhabilitation du paysage comme catégorie de l'action publique. Ainsi les thèses d'un Alain Roger, largement reprises par ceux qui ont œuvré sur le terrain du « projet de paysage » et des politiques en la matière, ont-elles pu contribuer en France, dans les années 1980 et 1990, à une re-légitimation des fondements hérités de l'action en ce domaine, en revêtant de nouveaux oripeaux les normes d'appréciation du paysage inscrit dans les codes du pittoresque. À l'heure d'une extension proclamée (dans la loi «paysage » de 1993 notamment) à l'ensemble du territoire du souci de la

21 . Une critique de l'assignation du paysage à un art et un sentiment esthétique épuré, autonome vis-à-vis de la connaissance est proposée par J.-M. Besse dans : « La physionomie du paysage d'Alexandre de Humboldt à Paul Vidal-de-La-Blache », dans J.-M. BESSE, 2000b. p. 97 sq. Cf. aussi BRIFFAUD, 2008. 
qualité du paysage, l'« artialisation » de Roger restaurait non seulement l'autorité en ce domaine d'une expertise traditionnelle, mais elle donnait caution à la « mise en pittoresque » de nouveaux territoires, jusqu'ici demeurés en dehors de la juridiction des esthètes patentés.

C'est relativement à ce même contexte sociétal et politique qu'il convient d'apprécier, pour partie au moins, l'apport des thèses de K. Olwig sur les origines $\mathrm{du}$ paysage. Autant les théories paysagères d'Alain Roger servait la restauration de normes anciennes de l'action, autant celles d'Olwig se prêtent à être mobilisées par ceux qui ressentent la nécessité d'une profonde redéfinition du paysage comme catégorie de l'action publique. Ces thèses sont formulées au moment précis où émerge, en Occident, une philosophie politique alternative, en ce domaine, à celle qu'ordonne le vieux paradigme du pittoresque. L'élaboration de la Convention européenne du paysage, signée en Florence en 2000, est un marqueur de ce tournant, qui conduit à des politiques référées non plus seulement aux évaluations d'un spectateur extérieur aux territoires concernés, mais aussi aux «perceptions des populations» qui habitent et exploitent ces territoires ${ }^{22}$. Ce nouveau paysage de l'action, qui dans les textes comme dans les pratiques se superpose à l'ancien plus qu'il ne l'abolit, flotte quelque part entre ce que produit l'acteur et ce que voit l'observateur, réalité tangible et représentation idéelle s'imbriquant l'une dans l'autre et se fondant dans ce que l'on appelle volontiers, désormais, le « paysage de l'habitant». Le récit d'Olwig est devenu aujourd'hui la référence par excellence de tout ceux — chercheurs comme acteurs de terrain qui se réclament de ce nouvel objet. Autour de lui se crée un communauté de pensée paysagère, qui transcende les frontières nationales; surpassant l'opposition entre écoles de pensée anglo-américaines et franco-méditerranéennes.

Le ralliement au récit d'Olwig va de pair avec une généralisation de ses conclusions à l'ensemble de l'Europe. Dans un texte sur « Landscape, culture and modernity », publié en 2006, Denis Cosgrove lui-même, révisant ses premières théories, attribue à la fois à countryside, paysage, paesaggio, paisaje le sens originel de Landschaft, affirmant qu'ils désignent d'abord des relations collectives au pays (Cosgrove, 2006). Yves Luginbühl, corédacteur de la Convention européenne du paysage, adopte lui-même dans son dernier ouvrage la perspective d'Olwig, sans véritablement poser le problème de la pertinence de sa généralisation . La thèse d'Olwig, affirme-t-il, « confirme le rôle de l'acteur bien plus que du spectateur dans la production des paysages et rejoint la proposition [...] de donner au paysage le sens d'un projet d'aménagement partagé par les acteurs $»{ }^{23}$. Jean-Marc Besse a également noté le lien fort qui s'établit entre les thèses d'Olwig sur la «nature substantielle» et originelle du paysage et une (re)définition de l'action en la matière. «On pourrait, remarque-t-il, mettre en relation [cette] reconstruction historique et la notion de «projet local» dont

22. K. Olwig lui-même analyse la Convention européenne en fonction de sa propre approche du paysage dans Olwig, 2007. Cf. aussi sur les sous-bassements politiques de la théorie d'Olwig: Olwig et Mitchell, 2009.

23. Luginbühl, 2012. L'auteur ne pose pas la question de la conciliation de la thèse d'Olwig avec le résultat des recherches philologiques de Catherine Franceschi, qu'il expose néanmoins, en rappelant que «les sens premiers [des équivalents de paysage en Europe] varient donc le plus souvent autour de cette notion de spectacle du "pays" ». (Ibid., p. 50). 
Alberto Magnaghi s'est fait le champion en Italie dans ses analyses sur la « soutenabilité politique»»(Besse, 2012, p. 86). Besse note bien également l'opposition que porte le récit d'Olwig, mais plus généralement les théories angloaméricaines du paysage à un "paysage de l'État», identifié à un paysage spectatorial et scénographique, au profit d'un paysage de la communauté "régie et régulée par un ensemble de coutumes et qui cherche à les préserver » (Ibid.).

Parmi les récits contemporains sur les origines du paysage, celui d'Olwig est le premier à véritablement ouvrir la perspective d'une véritable reconstruction de l'objet paysage, sur les ruines de celle qu'avait engagée l'époque romantique. Le Cosgrove des années 1980 s'intéressait aux effets sociaux, culturels et spatiaux du mode paysager d'appréhension des réalités géographiques, mais ne proposait pas de voir dans le paysage autre chose que ce regard institué par l'avènement du capitalisme et déterminée par cette origine. La perspective ontologique proposée par un Descola ou un Berque, dans la mesure même où elle traite l'appréhension paysagère $\mathrm{du}$ monde environnant en discriminant anthropologique, exclut également toute redéfinition de la notion. Quant aux récits gombrichiens (auxquels ces deux derniers auteurs empruntent assez largement), et en particulier à celui de Roger, ils ne relèvent pas réellement d'une construction, mais de la réification d'une objectivation héritée du paysage, qui néanmoins tire ce dernier en direction d'un «pur» sentiment esthétique auquel les romantiques ne l'avait pas associé. Le récit d'Olwig en revanche, qui assigne au paysage une origine nouvelle, se présente bel et bien comme l'opérateur d'une nouvelle objectivation, renversant une conception héritée du paysage, fondée sur un récit inexact des conditions de son avènement.

On peut légitimement s'interroger sur le nouveau paysage que dessine cet effort de ré-objectivation. Déplacé à grand renfort d'exclamations antispectatoriales en direction du «lieu » et de l'« espace vécu », ce paysage ne vientil pas en définitive occuper une place déjà prise par d'autres concepts, voire par des politiques qui les mobilisent? Mais ce qui pousse à s'interroger est d'abord le moyen pour le moins éculé que cette ré-objectivation mobilise. En recourant à la preuve par l'origine, celle-ci demeure hélas fidèle à une vision essentialiste du paysage, qui fait obstacle à la transformation de celui-ci en un véritable concept, aux définitions ouvertes et discutables.

\section{En finir avec la quête des origines et reconstruire l'histoire du paysage}

Constatons pour conclure que le recours à des grands récits paysagers de fondation a constitué ces dernières décennies un réel obstacle au développement d'une véritable histoire des relations des sociétés à leurs environnements perceptibles. La volonté d'objectiver ainsi le paysage a contribué à fausser la vision que l'on se fait de l'attitude spectatoriale des occidentaux vis-à-vis de ces environnements, en poussant à la regarder comme relevant tout uniment, en particulier à partir de l'époque moderne, d'un mode paysager de regard. On a ainsi installé de la continuité historique là où il n'y en avait pas en réalité.

Il faut par conséquent réinterroger sur de nouvelles bases cette attitude spectatoriale, ses conditions de possibilité, les valeurs qu'on lui associe à différentes époques, les règles que l'on impose aux regardeurs. On doit le faire en 
demeurant attentif aux formes plurielles que revêt cette attitude et sans donc l'associer a priori à une vision "paysagère " aux caractéristiques prédéfinies. Il importe également au plus haut point de cesser de confondre l'environnement perceptible avec une "nature » face à laquelle l'observateur se trouverait fatalement placé. La question fondamentale est précisément de savoir ce que ce dernier saisit - ce que cet environnement représente à ses yeux. Elle est de savoir, notamment, si la "nature " elle-même est présente dans ce spectacle du monde extérieur et à quelle condition elle en devient l'hôte. Il s'agit en un mot de savoir comment le regard et ce qu'il appréhende se co-construisent, et par conséquent de sortir d'une vision figée de la relation spectatoriale, qui place le spectateur devant un donné perceptif toujours identique à lui-même et qu'il serait simplement capable, ou non, de voir et d'apprécier.

Il s'agit bien aussi d'interroger le ou les moments où ce spectacle du monde extérieur devient paysage, est objectifié comme tel, c'est-à-dire précisément ce que les grands récits paysagers, qui ne s'intéressent qu'à une « réalité » de la perception considérée a priori comme indépendante de sa conceptualisation, n'interrogent pas eux-mêmes. Or, on peut, on l'a vu, faire l'hypothèse que ce que l'observateur occidental perçoit ne devient que très tardivement un "paysage » objectivable comme tel. Affirmer cela n'a pas pour conséquence de retirer à d'autres époques et à d'autres sociétés la relation spectatoriale à leur environnement, ni le « sentiment de la nature ».

Il se pourrait que la spécificité d'un « regard » occidental, que l'on a voulu associer intimement à une appréhension paysagère de la nature, ressorte plus clairement d'un tel déplacement de la problématique. Ne pas placer a priori le spectateur occidental dans l'alternative entre vision et non vision paysagère permet d'appréhender la construction et les mutations de son regard sous l'angle d'une dialectique aux configurations datables de l'attrait pour les spectacles du monde extérieur et des résistances à cette extravertion. Cette dialectique pourrait bien être le moteur principal, dans la très longue durée - depuis au moins les premiers siècles de l'ère chrétienne jusqu'à une époque très récente - de l'histoire du rapport des sociétés occidentales à l'environnement perceptible. Pendant des siècles, en d'autres termes, la relation spectatoriale au monde extérieur s'est construite dans le dépassement ou le contournement de ce qui l'entrave, la rend suspecte, ou simplement inutile — croyances religieuses, règles morales, normes épistémologiques et esthétiques... C'est là, dans ce dépassement, que se construit, on peut le penser, toute la richesse, la complexité et la pluralité historique des regards et des représentations associées à cette posture.

Il est possible que le «moment» du paysage ne soit qu'une parenthèse, d'assez courte durée, dans l'histoire des relations de l'Occident à ses mondes environnants. C'est là l'hypothèse que proposait déjà, au début des années 1980, Denis Cosgrove et quelques autres auteurs évoquant la "mort du paysage » (Dagognet, 1982). L'idée mérite sans aucun doute d'être réexaminée. Relativement au point de vue qui vient d'être proposé, ce moment du " paysage » pourrait bien être celui sur lequel s'étend le règne, aujourd'hui contesté, de la figure de l'observateur, telle que construite aux temps romantiques. 
Ne faudrait-il pas considérer dès lors que les deux grandes périodes où fleurissent les théories sur les origines du sentiment paysager encadrent ce court moment du paysage et nous parlent, pour les unes d'une émergence et, pour les autres, d'une crise ou d'une agonie, que masque et révèle à la fois l'effervescence théorique de ces dernières décennies?

Bordeaux, juillet 2014

\section{Références bibliographiques}

ALPERS, S. 1990. L'Art de dépeindre: la peinture hollandaise au XVIIe siècle. Gallimard.

ANDREWS, M. 1990. The Search for the Picturesque: Landscape Aesthetics and Tourism in Britain, 1760-1800. Scolar P., Aldershot.

BARIDON, Michel. 2000. Le Jardin paysager anglais au dix-huitième siècle. Dijon : Editions universitaires de Dijon.

BARRELL, John. 1980. The Dark Side of the Landscape. The Rural Poor in English Painting 1730-1840. Cambridge : Cambridge University Press.

BERMINGHAM, Ann. 1989. Landscape and ideology. The english rural tradition, 1740-1860. Standford : Standford University Press.

BERQUE, Augustin. 1995. Les raisons du paysage, de la Chine antique aux environnements de synthèse. Paris : Hazan; et surtout :

BERQUE, Augustin. 2008. "Cosmophanie et paysage moderne ». Dans Aline BERGE et Michel COLLOT (éd.). Paysage et modernité. Bruxelles : Ousia, p. 43-65.

BESSE, Jean-Marc. 2000a. « J.B. Jackson et la géographie humaine : les débuts de la revue Landscape ». Le Visiteur (n ${ }^{\circ}$ 5): p. 106-129.

BESSE, Jean-Marc. 2000b.Voir la terre. Six essais sur le paysage et la géographie, Arles : Actes Sud.

BESSE, Jean-Marc. 2003. «Le paysage, entre le politique et le vernaculaire. Réflexions à partir de John Brinckerhoff Jackson ». ARCES (n $\left.{ }^{\circ} 6\right)$ : p. 9-27.

BESSE, Jean-Marc. 2012. «Du point de vue de l'espace. Quelle histoire du paysage européen ». dans Paysages européens et mondialisation. Seyssel : Champ Vallon.

BRIFFAUD Serge. 2013. Le paysage, le regard et le temps. Enquête historique et géographique sur l'empaysagement des espaces et des sociétés, Mémoire pur l'Habilitation à Diriger des Recherches, Université de Bordeaux Montaigne. 
BRIFFAUD, S. 1994. Naissance d'un paysage. La montagne pyrénéenne à la croisée des regards, $X V I^{\circ}-X I X^{\circ}$ siècle. Université de Toulouse II et Archives départementales des Hautes-Pyrénées, Toulouse et Tarbes.

BRIFFAUD, Serge. 2006. « Le temps du paysage. Alexandre de Humboldt et la géohistoire du sentiment de la nature. ». Dans Géographies plurielles. Les sciences géographiques au moment de l'émergence des sciences humaines (17501850), Isabelle LABOULAIS, Hélène BLAIS (éd.). Paris : L'Harmattan, p. 275297.

BRIFFAUD, Serge. 2007. "Actualité du paysage humboldtien». Dans Gilles BERTRAND et Alain GUYOT (dir.), Des " passeurs » entre science, histoire et littérature. Contribution à l'étude de la construction des savoirs, Grenoble: ELLUG, 2011, p. 151-165.

BRIFFAUD, Serge. 2008. «Le paysage, les sciences et l'esthétique. Quelques constats et réflexions ». Dans Georges BERTRAND et Serge BRIFFAUD (éd.), Le paysage : retour d'expériences entre recherche et projet, actes du colloque d'Arthous, 9-10 octobre 2008, Mont-de-Marsan et Arthous : Conseil général des Landes, 2011, p. 162-169.

BRUNON Hervé. 2006. «L'essor artistique et la fabrique culturelle du paysage à la Renaissance. Réflexions à propos de recherches récentes ». Studiolo. Revue d'histoire de l'art de l'Académie de France à Rome 4, p. 261-290.

BRUNON Hervé. 2011. «Paysage et Histoire de l'art. Un «geographic turn »? ». Revue de l'art, $\mathrm{n}^{\circ}$ 173-3 p. 13-19.

CARUS, Carl Gustav et FRIEDRICH, Caspar David. 2003 [1831]. De la peinture de paysage dans l'Allemagne romantique. Paris : Klincksieck.

COPLEY, Stephen, GARSIDE, Peter (sous la dir. de). 1994. The politics of the picturesque. Literature, landscape and aesthetics since 1770. Cambridge : Cambrige Université Press.

CORBIN, Alain. 1988. Le territoire du vide. L'Occident et le désir du rivage, 1740-1840. Paris : Aubier.

COSGROVE, Denis. 1984. Social Formation and Symbolic Landscape. University of Wisconsin Press.

COSGROVE, Denis. 1985. «Prospect, Perspective and the Evolution of the Landscape Idea ». Transactions of the Institute of British Geographers 10 (1), p. 45-62.

COSGROVE, Denis. 1993. The Palladian landscape. Geographical Change and its Cultural Representations in Sixteenth-Century Italy, Leicester : Leicester University Press.

COSGROVE, Denis. 2007. Geography and vision. Londres : Y.B. Tauris.

CRARY, Jonathan. 1994. L'art de l'observateur. Vision et modernité au XIXe siècle. Nîmes: Editions Jacqueline Chambon.

DAGOGNET, François (dir.). 1982. Mort du paysage ?: Philosophie et esthétique du paysage. Seyssel : Editions Champ Vallon. 
DANIELS, Stephen. 1999. Humphrey Repton, Landscape Gardening and the Geography of Georgian England. Yale University Press.

DECULTOT, Elisabeth. 1996. Peindre le paysage : discours théorique et renouveau pictural dans le romantisme allemand. Tusson : éd. du Lérot.

DECULTOT, Élisabeth. 2009. « Tout converge vers le paysage. La notion de Landschafterey chez Philipp Otto Runge et la question des frontières entre genres picturaux ». Dans Romantisme et frontières. Alain Muzelle (éd.). Nancy : CEGIL. p. 117-130.

DELIGNE, Alain. 2003. La terre qui vit : peinture et savoirs chez Carl Gustav Carus (1789-1869). Villeneuve d'Ascq : Presses Universitaires du Septentrion.

DESCOLA, Philippe (éd.). 2011. La fabrique des images. Visions du monde et formes de la représentation. Paris : Musée du Quai Branly et Somogy, p. 73-97

DESCOLA, Philippe. 2005. Par-delà nature et culture. Paris : Gallimard.

ELIAS, Norbert.1985 [1933]. La société de cour. Paris : Flammarion.

Ernst H. GOMBRICH. 1983 [1953]. Renaissance Artistic Theory and the Development of Landscape Painting, in Gazette des Beaux-Arts, XLI, JanuaryJune 1953, p. 335-360 ; repris sous le titre The Renaissance Theory of Art and the Rise of Landscape, in Norm and Form : Studies in the Art of the Renaissance, London, 1966, p. 107-121, trad. fr. La théorie artistique de la Renaissance et l'essor du paysage. Dans L'Écologie des images, Paris : Flammarion, 1983, p. 1543.

FOUCAULT, Michel. 1963. Naissance de la clinique. Paris: Presses Universitaires de France.

FOUCAULT, Michel. 1966. Les mots et les choses: une archéologie des sciences humaines. Paris : Gallimard.

HARRIS, Dianne. 1999. "The postmodernization of Landscape. A critical Historiography ». The Journal of the Society of Architectural Historians, vol. 58, $\mathrm{n}^{\circ}$ 3, Architectural History, 1999/2000. (Sept.), p. 434-443.

HARTOG, François. 2005. Évidence de l'histoire : Ce que voient les historiens. Paris : Éditions de l'École des hautes études en sciences sociales.

HOOZEE, R. 2007. British Vision: Observation and Imagination in British Art, 1750-1950. Mercatorfonds.

HUMBOLDT, Alexandre de. 2000. Cosmos : essai d'une description physique du monde. Thizy : Utz, 2 vol. Rééd. de l'édition de 1846-1851. Paris : Gide et Baudry.

HUNT, John Dixton. 1992. Gardens and the Picturesque : Studies in the History of Landscape Architecture. Massachusetts : MIT Press.

J.M BESSE. 2009. Le goût du monde : Exercices de paysage. Arles : Actes Sud.

JACKSON, John B. 2003. À la découverte du paysage vernaculaire. Arles : Actes Sud (traduction de Discovering the vernacular landscape, lére éd. Américaine : 1983). 
JACKSON, John B. 2005. De la nécessité des ruines et autres sujets. Paris : Editions du Linteau (traduction de The Necessity for ruins and other topics, 1ère édition américaine : 1980).

JACKSON, John Brinckerhoff. 2005 [1979]. « Le paysage comme théâtre ». Dans De la nécessité des ruines et autres sujets. ouvr. cité, p. 109-120

JAKOB Michael. 2011. Cent paysages. Expositions d'un genre, Genève : Infolio.

KLONK, Charlotte. 1996. Science and the Perception of Nature: British Landscape Art in the Late Eighteenth and Early Nineteenth Centuries. Yale University Press for The Paul Mellon Centre for Studies in British Art.

LEE, R.W. 1991. Ut pictura poesis: humanisme et théorie de la peinture, XVeXVIIIe siècles. Paris : Macula.

LUGINBÜHL, Y. 2007. Pour un paysage du paysage. Economie rurale, 297298(1-2), 23-37.

LUGINBÜHL, Y. 2012. La mise en scène du monde. Construction du paysage européen. Paris : CNRS Editions.

MARIN, Louis. 1993. Des pouvoirs de l'image. Gloses. Paris : Éditions du Seuil, p. 11.

MARTINET, Marie-Madeleine. 1980. Art et nature en Grande-Bretagne : de l'harmonie classique au pittoresque du premier romantisme, 17e-18e siècles. Paris : Aubier-Montaigne.

MILANI, R. 1996. Il pittoresco: l'evoluzione del Gusto tra classico e romantico. Ed. Laterza.

MITCHELL W.J. Thomas (éd.). 1994a. Landscape and Power. Chicago : University of Chicago Press.

MITCHELL, W.J.T. 1994b. «Imperial Landscape ». Dans Landscape and power. MITCHELL, W.J.T. (éd.). Chicago : University of Chicago Press.

OLWIG, Kenneth R.; MITCHELL, Donald. 2009. Justice, Power and the Political Landscape. Londres : Routledge.

OLWIG, Kenneth R. 1996. Recovering the Substantive Nature of Landscape. Annals of the Association of American Geographers 86 (4): 630-653

OLWIG, Kenneth R. 2002. Landscape, Nature and the Body Politic. Madison : University of Visconsin Press.

OLWIG, Kenneth R. 2007. The practice of landscape 'conventions' and the just landscape: the case of the European Landscape Convention. Landscape research, vol. $32, \mathrm{n}^{\circ} 5$, p. $579-594$.

PILES, Roger de. 1708. Cours de peinture par principes. Paris : J. Estienne ; rééd. 1989. Paris : Gallimard.

RECHT, Roland. 1989. La Lettre de Humboldt : du jardin paysager au daguerréotype. Paris : Bourgois. 
RILKE, Rainer Maria. 1966. «Sur le paysage » [1902]. Oeuvres 1. Prose. Paris : Le Seuil, p. 369-390.

ROGER, Alain. 1997. Court traité du paysage. Paris : Gallimard.

SAUER, C.O. 1925. The Morphology of Landscape, by Carl O. Sauer. California University Press.

TISSIER, Jean-Louis. 2003. "Paysage». Dictionnaire de la géographie et de l'espace des sociétés. Jacques Lévy, Michel Lussault (éds.). Paris : Belin. p. 697701.

TURRI, Eugenio. 1998. Il paesaggio come teatro. Venezia : Marsilio.

WALTER, François. 2004. Les figures paysagères de la nation. Territoire et paysage en Europe (16 ${ }^{\circ}-20^{\circ}$ siècle). Paris : Editions de l'EHESS.

WAT, Pierre. 1998. Naissance de l'art romantique: peinture et théorie de l'imitation en Allemagne et en Angleterre. Paris : Flammarion.

WILLIAMS, Raymond. 1977. "Plaisantes perspectives ». Actes de la recherche en sciences sociales $\mathrm{n}^{\circ} 17-18$, p. 29-36.

WILLIAMSON, Tom. 1995. Polite landscapes. Gardens and society in eighteenth century England. Londres : Sutton Publishing. 